\title{
Hydrogeochemical Model and Water Quality of Groundwater in the Granito-Basaltic Fractured Rock Aquiferous Formations in Bafoussam, West Region-Cameroon
}

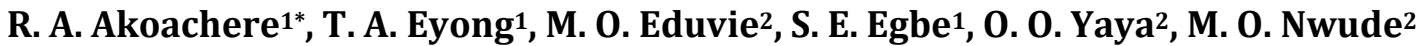 \\ ${ }^{1}$ Department of Geology, University of Buea, Buea, Cameroon \\ ${ }^{2}$ National Water Resources Institute, Kaduna, Nigeria \\ Email: *r.akoachere@ubuea.cm
}

How to cite this paper: Akoachere, R.A., Eyong, T.A., Eduvie, M.O., Egbe, S.E., Yaya, O.O. and Nwude, M.O. (2018) Hydrogeochemical Model and Water Quality of Groundwater in the Granito-Basaltic Fractured Rock Aquiferous Formations in Bafoussam, West Region-Cameroon. Journal of Water Resource and Protection, 10, 1148-1174.

https://doi.org/10.4236/jwarp.2018.1011068

Received: September 24, 2018

Accepted: November 24, 2018

Published: November 27, 2018

Copyright (C) 2018 by authors and Scientific Research Publishing Inc. This work is licensed under the Creative Commons Attribution International License (CC BY 4.0).

http://creativecommons.org/licenses/by/4.0/

\section{(c) (i) Open Access}

\begin{abstract}
This study determined the hydrogeochemical model of groundwater and groundwater domestic-agro-industrial quality in Bafoussam using hydrogeochemical tools and physicochemical parameters: Ionic ratios, Gibbs diagrams, Piper diagrams, Durov diagrams and water quality indices. From physicochemical parameters; $\mathrm{pH}$ ranged from, 4.47 - 7.84; EC, 10 - $820 \mu \mathrm{S} / \mathrm{cm}$; Temperature, $22.3^{\circ} \mathrm{C}-29.5^{\circ} \mathrm{C}$ and TDS, $6.7-549.4 \mathrm{mg} / \mathrm{L}$. The major ions fell below WHO acceptable limits. The sequences of major ionic abundance are: $\mathrm{Ca}^{2+}>\mathrm{Mg}^{2+}>\mathrm{K}^{+}>\mathrm{Na}^{+}>\mathrm{NH}_{4}^{+}, \mathrm{HCO}_{3}^{-}>\mathrm{Cl}^{-}>\mathrm{SO}_{4}^{2-}>\mathrm{NO}_{3}>\mathrm{HPO}_{4}^{2-}$. Recharge by atmospheric precipitation, ion-exchange and simple dissolution processes are responsible for groundwater character, ionic content resulted from ion exchange and rock-weathering. Water types are $\mathrm{Ca}-\mathrm{HCO}_{3}$ and $\mathrm{Ca}-\mathrm{Cl}$ Hydrogeochemical facies are $\mathrm{Ca}-\mathrm{Mg}-\mathrm{Cl}-\mathrm{SO}_{4}$ and $\mathrm{Ca}-\mathrm{Mg}-\mathrm{HCO}_{3}$. Domestic water quality was determined by use of $\mathrm{pH}$, electrical Conductivity EC, total dissolved solids TDS, total Hardness $\mathrm{H}_{\mathrm{T}}$ and water quality index WQI. WQI values ranged from $0-42.09$ and $\mathrm{H}_{\mathrm{T}} 67.89-339.01$ indicating that water is of good domestic quality. Agro-industrial suitability of groundwater was determined using, sodium adsorption ratio SAR, permeability index PI, Magnesium adsorption ratio MAR, percent sodium \%Na, Kelly's ratio KR and Residual sodium carbonate RSC and Wilcox diagram; From irrigational water suitability parameters, SAR values ranged from $0.01-005 ; \% \mathrm{Na} 3.69$ 15.50; KR 0.005 - 0.023; PI 1.04 - 67.98; MAR 2.89 - 55.27; RSC -5.22 to -0.44 and Wilcox diagram indicate that inorganic groundwater content in the study area is excellent-good for irrigation; this is of significance since Bafoussam a major agroindustrial zone in Cameroon and Central Africa is in the process of developing large scaled irrigation based agricultural projects dependent on
\end{abstract}


use of surface and groundwater. Recharge from precipitation exchanges ions with the weathered country rocks and mixes with regional flow in a generally south-east north-westerly direction by piston flow in the granito-basaltic aquiferous formations in Bafoussam. There is need for detailed studies to determine aquifer characteristics: permeability, transmissivity and storativity, vertical-lateral regional extent of aquifer boundaries, groundwater pollution potentials for biological, organic and trace metals.

\section{Keywords}

Hydrogeochemical-Model, Water-Quality, Fractured-Rock-Aquifer, Bafoussam, Cameroon

\section{Introduction}

Bafoussam situates between latitude $5.42-5.60 \mathrm{~N}$ and longitude $10.38-10.48 \mathrm{E}$ is the capital, and largest city of the Western Region of Cameroon in the Bamboutous Mountains. Bafoussam has a maximum elevation of about $1450 \mathrm{~m}$ a.m.s.l, covering a surface area of about $450 \mathrm{~km}^{2}$. It is bordered geographically to the south by the Littoral Region, to the east by the Centre Region, to the north by the North West and Adamawa Regions and to the west by the South west region. Bafoussam is a conurbation of seven villages: Bamenzi, Banengo, Ndiangdam, Ndiangsouoh, Ndiangbou, Toukouop and Njoueng (Figure 1). It has a population of over 500,000 inhabitants, with a population density of 512 people per square kilometer [1]. The climate is constantly cool and has temperatures oscillating between $15^{\circ} \mathrm{C}-28^{\circ} \mathrm{C}$. The amount of precipitation for a year in $\mathrm{Ba}-$ foussam is about $20,000 \mathrm{~mm}$. The city does not have many rivers. Mainly, it consists of several streams with variable discharge rates, depending on the season. The main river in the study area is the River Noun, which acts as a boundary with the Kouoptamo municipality. The soil is primarily composed of alluvio-colluvial material that resulted from the weathering of igneous and volcanic rocks. As one of the largest cities in Cameroon, the main economic activity carried out in Bafoussam is agriculture. The inhabitants plant cash crops such as coffee, cocoa, oil palm, peanuts, banana and other food crops like tomatoes, beans, corn, Irish potatoes, yam, okra, cassava and cabbage. Poultry farming is also well developed here, with many poultry farmers involved in table birds and egg production.

The need to provide water of good quantity and quality to humans is of great concern as over $80 \%$ of waterborne illnesses are attributed to poor water quality [2]. This makes it important to characterize the quality of groundwater through the determination of physicochemical content of groundwater, establish a hydrogeochemical assessment in order to ensure sustainable supply of water resources and for effective habitat conservation plans in Bafoussam.

With the increasing population and creation of large scale agroindustrial farms, there will be an increased pressure on the water quantity and quality in 


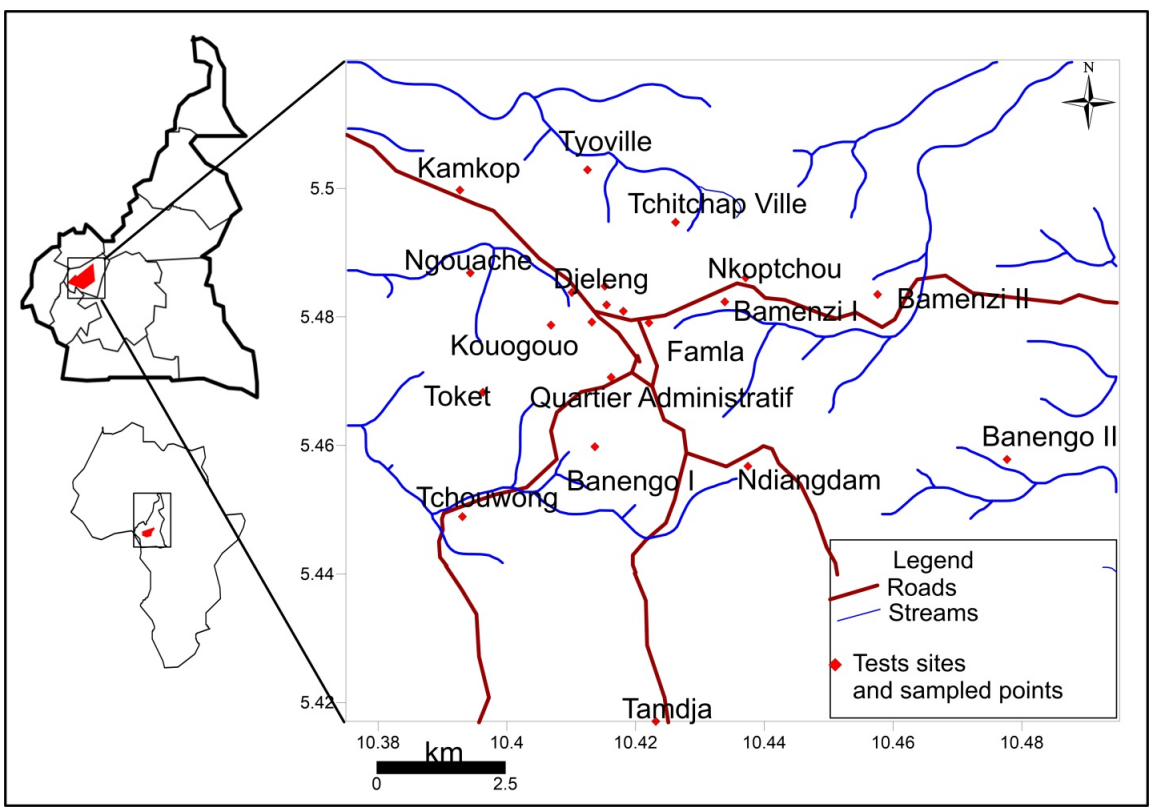

Figure 1. Location of tested wells; measurement and sampled points.

Bafoussam. Knowledge of the hydrogeochemistry and water quality of the groundwater in Bafoussam is thus a necessity. Also, in all future planning on urbanization and development of this city, groundwater quantity and quality must be defining parameters. This study to evaluate the hydrogeochemistry, drinking and agricultural groundwater suitability is as a baseline to provide a major tool for management and protection of the groundwater resources in Bafoussam.

\section{Geologic Setting of the Study Area}

Bafoussam in west region of Cameroon is a contact zone of the Central African Orogenic Belt, located along the southern extension of the Central Cameroon Shear Zone and the Cameroon Volcanic Line. It is dominated by granitoids which belong to the Pan-African syn- to post-collisional post-650 Ma group [3]. Syenogranites are predominant with alkali-feldspar granite, monzogranite, quartz-monzonite and quartz-monzodiorite. Four granitoid suites, biotite granitoids and deformed biotite granitoids with amphibole, megafeldspar granitoids with megacrysts and two-mica granitoids with primary muscovite and igneous garnet are distinguished. The granites can be assigned to high-K calc-alkalic to shoshonitic series. The partly shoshonitic biotite granitoids are metaluminous to weakly peraluminous and can be labelled as a highly fractionated I-type suite. The megafeldspar granitoids are weakly peraluminous with I-type character whereas the two-mica granitoids are weakly to strongly peraluminous and belong to an S-type suite [3]. The CVL is responsible for the vast igneous rock formations found in Bafoussam and the shearing caused by the CCSZ and the displacement of the Cameroon neoproterozoic orogenic belt is the main driving force that led to the deformation of the rocks.

Neoproteroizoic Orogenic belt is the main driving force that led to the defor- 
mation of the rocks. A vast majority of the study area is covered by tertiary basalts that outcrop in various locations and form structures such as columnar basalts at some outcrops. Columnar basalts are structures that are usually hexagonal in shape and separated by joints and fractures formed when the rock contracted, most often during cooling.

Granitoid rocks are also abundant in the study area where they occur dominantly on hill slopes or along river cuts in the northwestern parts of the city. Two types are observed in this area; the biotite granitoids and mega feldspar granitoids (Figure 2). Some of these granitoids show preferred mineral alignment, indicating that they had undergone some deformation after their formation. Columnar basalts occur extensively in the area and are extracted for construction purposes in Figure 3. A 3-D model of the fractured-rock aquiferous formations in Bafoussam from the field geological survey is shown in Figure 4.

\section{Materials and Methods}

\subsection{Materials}

The field materials and equipment used in the study are listed in Table 1. The equipment were calibrated and used according to manufacturer's specifications.

\subsection{Methods}

A reconnaissance survey was carried out to identify wells, springs and streams in February 2018 [4]. 119 dug wells were measured/tested insitu for: coordinates of wells, Surface elevation, static water level, wells depths, well diameter, Electrical conductivity (EC), $\mathrm{pH}$, Total dissolved solids (TDS) and Temperature $\left({ }^{\circ} \mathrm{C}\right)$.

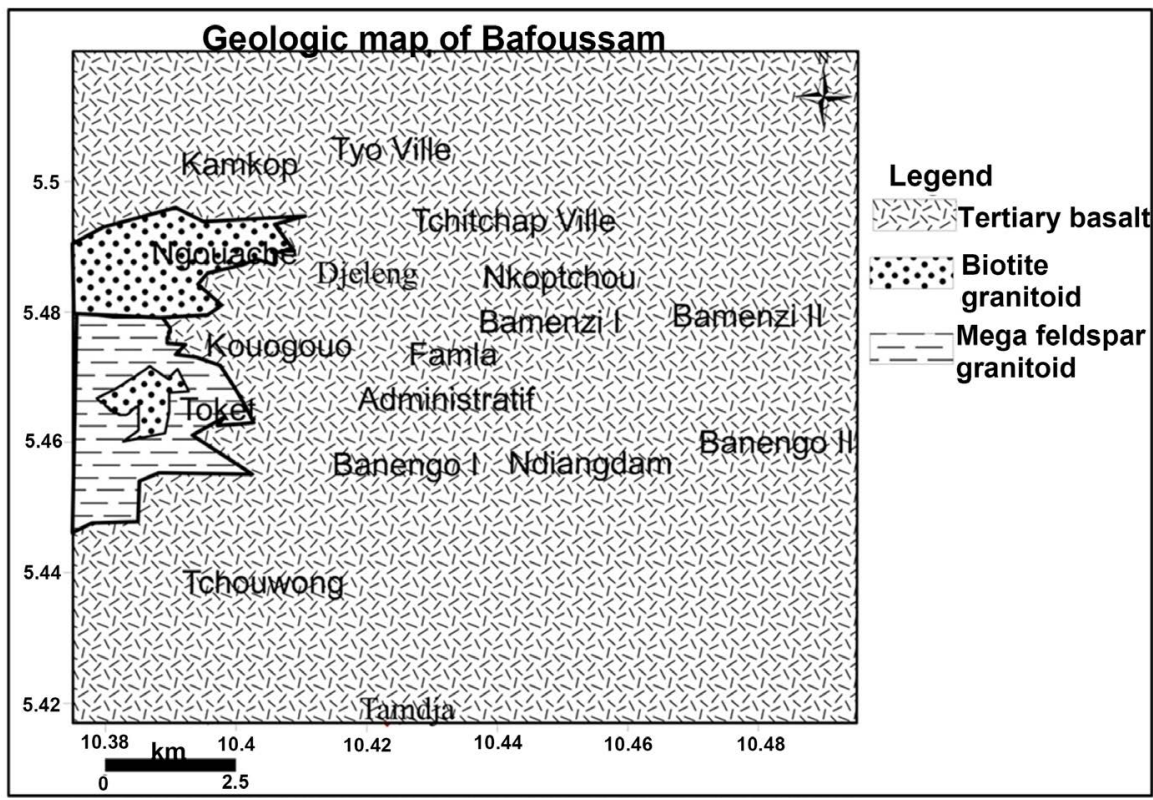

Figure 2. Geology of Bafoussam; composed of tertiary basalts, biotite granitoids and mega feldspar granitoids. The tertiary basalts are columnar with column diameters up to 3.6 meters. 


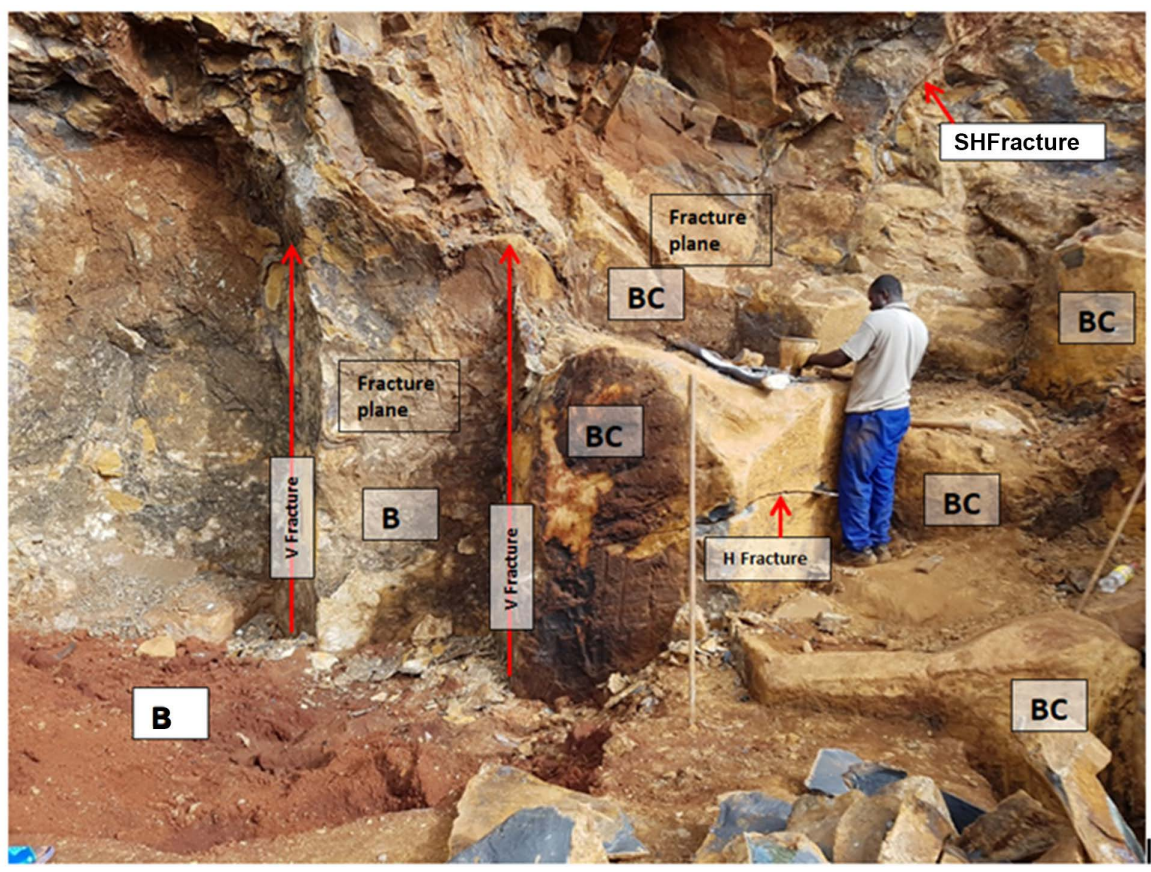

Figure 3. Fractured rock columnar tertiary basalt formation, outcrop at Tamja Quarry Bafoussam: $\mathrm{BC}=$ Basalt column; $\mathrm{H}=$ Horizontal; $\mathrm{SH}=$ Sub horizontal; $\mathrm{V}=$ Vertical.

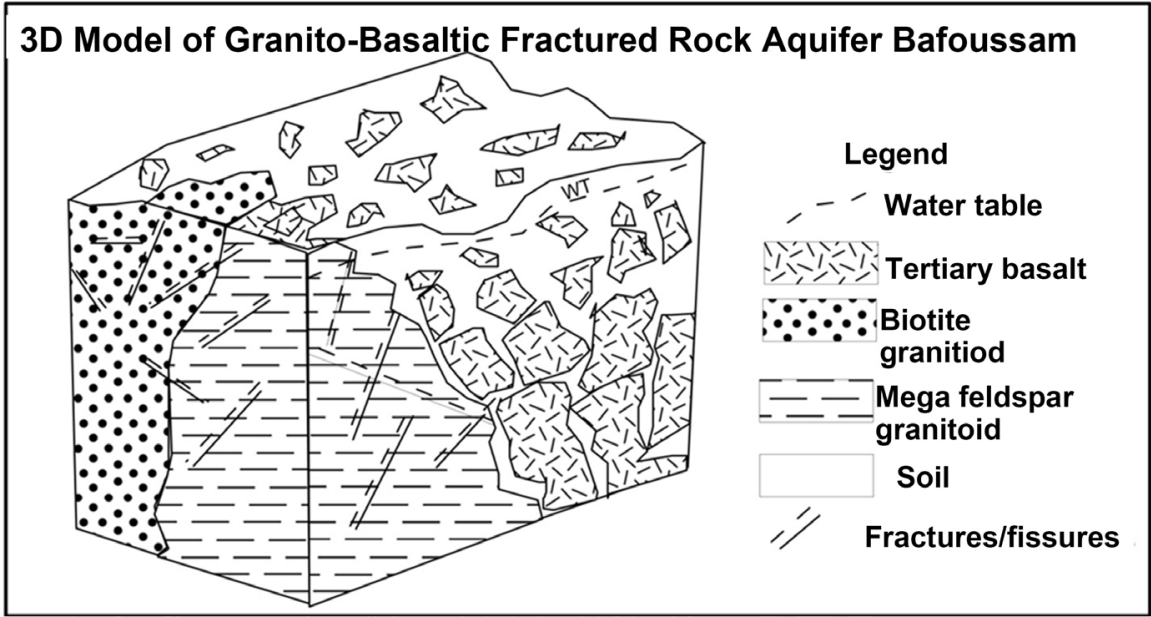

Figure 4. 3D model of granito-basaltic aquifer, Bafoussam.

Twenty three (23) groundwater samples were collected in high density polyethylene (HPDE) $500 \mathrm{ml}$ bottles sealed and sent to the laboratory based on sampling protocols [5] [6] and using standard methods [7], to analyze for:

1) Major cations in $\mathrm{mg} / \mathrm{l}: \mathrm{Ca}^{2+}, \mathrm{Mg}^{2+}, \mathrm{Na}^{+}, \mathrm{K}^{+}$and $\mathrm{NH}_{4}^{+}$.

2) Major anions in mg/l: $\mathrm{HCO}_{3}^{-}, \mathrm{Cl}^{-}, \mathrm{SO}_{4}^{2-}, \mathrm{HPO}_{4}^{2-}$ and $\mathrm{NO}_{3}^{-}$.

Ionic ratio of indicative elements is a useful hydrogeochemical tool to identify source rock of ions and formation contribution to solute hydrogeochemistry [8]. These were used in this study.

Gibbs Diagram is a plot of $\mathrm{Na}^{+} /\left(\mathrm{Na}^{+}+\mathrm{HCO}_{3}^{-} \mathrm{Ca}^{2+}\right)$ and $\mathrm{Cl}^{-} /\left(\mathrm{Cl}+\mathrm{HCO}_{3}^{-}\right)$as a function of TDS are widely employed to determine the sources of dissolved 
Table 1. Field equipment, specifications and functions.

\begin{tabular}{ccc}
\hline Equipment/Softwares & Specifications & Functions \\
\hline Bike & Commercial bikes (Bensikin) & Transport fieldworkers to well site \\
GPS & GARMIN GPSMAP 60CSx & Longitude, latitude and elevation of wells \\
EC Meter & HANNA HI 98304/HI98303 & To measure Electrical Conductivity of water \\
pH Meter & HANNA HI 98127/HI98107 & To measure pH of water \\
Water Level Indicator & Solinst Model $102 \mathrm{M}$ & To indicate static water levels of water in wells \\
Measuring Tape & Weighted measuring tape & Measurement of well diameter and depth \\
Digital Thermometer & Extech 39240 (-50 ${ }^{\circ} \mathrm{C}$ to $\left.200^{\circ} \mathrm{C}\right)$ & To measure temperature of water \\
Total Dissolved Solid meter & Hanna HI 96301 with ATC & To measure Total dissolved solids in water \\
Water sampler & Gallenkampf $1000 \mathrm{ml}$ & To collect well water sample from well \\
Sample bottles & Polystyrene $500 \mathrm{ml}$ & To hold sample for transmission to laboratory \\
ArcGIS & Version 10.1 & GIS Sampling/Tests location maps \\
Global Mapper & Version 15 & GIS Geolocation of wells \\
Surfer Golden Software & Version 12 & GIS Plotting, contouring spatial distribution \\
AqQA/Aquachem & Version 1.5 & Analysis/interpretation of water chemistry
\end{tabular}

geochemical constituents [9]. These plots reveal the relationships between water composition and the three main hydrogeochemical processes involved in ions acquisition; Atmospheric precipitation, rock weathering or evaporation crystallisation.

Pipers Diagram is a graphical representation of the chemistry of water sample on three fields; the cation ternary field with $\mathrm{Ca}, \mathrm{Mg}$ and $\mathrm{Na}+\mathrm{K}$ apices, the anion ternary field with $\mathrm{HCO}_{3}, \mathrm{SO}_{4}$ and $\mathrm{Cl}^{-}$apices. These two fields are projected onto a third diamond field [10]. The diamond field is a matrix transformation of the graph of the anions [sulphate + chloride] $/ \Sigma$ anions and cations $[\mathrm{Na}+\mathrm{K}] / \Sigma$ cations. This plot is a useful hydrogeochemical tool to compare water samples, determine water type and hydrogeochemical facies [11]. This has been used here for these purposes.

Durov diagram is a composite plot consisting of two ternary diagrams where the milliequivalent percentages of cations are plotted perpendicularly against those of anions; the sides of the triangles form a central rectangular binary plot of total cation vs. total anion concentrations [12]. These are divided into nine classes which give the hydrogeochemical processes determining the character of the water types in the aquiferous formation [11] [13].

WQI was calculated by adopting Weighted Arithmetical Index method considering thirteen water quality parameters $(\mathrm{pH}, \mathrm{EC}, \mathrm{TDS}$, total alkalinity, total hardness, $\mathrm{Ca}^{2+}, \mathrm{Mg}^{2+}, \mathrm{Na}^{+}, \mathrm{K}^{+}, \mathrm{Cl}^{-}, \mathrm{SO}_{4}^{2-}, \mathrm{NO}_{3}^{-}, \mathrm{NH}_{4}^{+}$) in order to assess the degree of groundwater contamination and suitability (Table 2).

For Agro-industrial suitability the following parameters were used; sodium adsorption ratio SAR, permeability index PI, Magnesium adsorption ratio MAR, 
Table 2. Indices used in the calculation of water quality and irrigation water quality.

\begin{tabular}{|c|c|c|}
\hline Index & Formula & Reference \\
\hline Percentage Sodium & $\% \mathrm{Na}=\frac{\mathrm{Na}^{+}+\mathrm{K}^{+}}{\mathrm{Na}^{+}+\mathrm{K}^{+}+\mathrm{Ca}^{2+}+\mathrm{Mg}^{2+}} \times 100$ & [14] \\
\hline Kelly Ratio & $K R=\frac{\mathrm{Na}^{+}}{\mathrm{Ca}^{2+}+\mathrm{Mg}^{2+}}$ & [15] \\
\hline $\begin{array}{c}\text { Magnesium Absorption } \\
\text { Ratio }\end{array}$ & $M A R=\left(\frac{\mathrm{Mg}^{2+}}{\mathrm{Mg}^{2+}+\mathrm{Ca}^{2+}}\right) \times 100$ & [16] \\
\hline Total Hardness & $\mathrm{TH}\left(\mathrm{CaCO}_{3}\right) \mathrm{mg} / \mathrm{L}=2.5 \mathrm{Ca}^{2+}+4.1 \mathrm{Mg}^{2+}$ & [17] \\
\hline Residual Sodium Carbonate & $R S C=\left(\mathrm{CO}_{3}+\mathrm{HCO}_{3}-(\mathrm{Ca}+\mathrm{Mg})\right)$ & [18] \\
\hline Sodium Absorption Ratio & $S A R=\frac{\mathrm{Na}}{\sqrt{\frac{\mathrm{Ca}+\mathrm{Mg}}{2}}}$ & [19] \\
\hline Permeability Index & $P I=\frac{\left((\mathrm{Na}+\mathrm{K})+\sqrt{\mathrm{HCO}_{3}}\right) * 100}{\mathrm{Ca}+\mathrm{Mg}+\mathrm{Na}+\mathrm{K}}$ & {$[20]$} \\
\hline Water Quality Index & $W Q I=\sum_{i=1}^{n} W_{i} q_{i}\left[\sum_{i=1}^{n} W_{i}\right]$ & [21] \\
\hline
\end{tabular}

percent sodium \%Na, Kelly's ratio KR and Residual sodium carbonate RSC and Wilcox diagram (Table 2).

The following softwares; Surfer 12, Global mapper 11 and AqQA 1.5 AGIS 10.3 were used for data presentation, interpretation and analysis.

\section{Results and Interpretation}

\subsection{Physicochemical Parameters}

The physicochemical parameters of groundwater for 119 wells in Bafoussam assessed are; EC, Temperature, $\mathrm{pH}$, and TDS (Table 3).

\subsubsection{Water Level Fluctuations}

Depth-to-static water level values $(\mathrm{m})$ of groundwater in Bafoussam ranged from: 0.48 - 18.21 (Figure 5). Static water levels are high and well depths are shallow. This indicates dug wells construction is relatively inexpensive and the technology available; a reason for the abundance of dug wells in Bafoussam. However these same reasons make the phreatic aquiferous formations vulnerable to pollution since some dug wells are poorly constructed.

\subsubsection{Groundwater Flow Contours}

Groundwater in Bafoussam flows from the central highest points at Talenga radiating outwards to the surrounding low lying areas; Tchouwong, Tamja, Depot G. and Ngouache in consonance with the topography: Piston flow (Figure 6).

\subsubsection{Temperature}

Temperature values of Bafoussam groundwater ranged from $22.3^{\circ} \mathrm{C}-29.5^{\circ} \mathrm{C}$ (Figure 7). These temperatures are just a few degrees below the air temperatures typical of phreatic aquifers. 
Table 3. Basic statistics of physicochemical parameters found in groundwater.

\begin{tabular}{ccccc}
\hline Parameters & Min & Max & Mean & Std. \\
\hline $\mathrm{T}\left({ }^{\circ} \mathrm{C}\right)$ & 22.30 & 29.50 & 24.94 & 1.25 \\
$\mathrm{pH}$ & 4.47 & 7.84 & 6.35 & 0.66 \\
$\mathrm{EC}(\mu \mathrm{S} / \mathrm{cm})$ & 10 & 820 & 268.24 & 200.16 \\
$\mathrm{TDS}(\mathrm{mg} / \mathrm{L})$ & 6.7 & 549.4 & 179.72 & 134.10 \\
\hline
\end{tabular}

\section{Depth to static water level, Bafoussam}

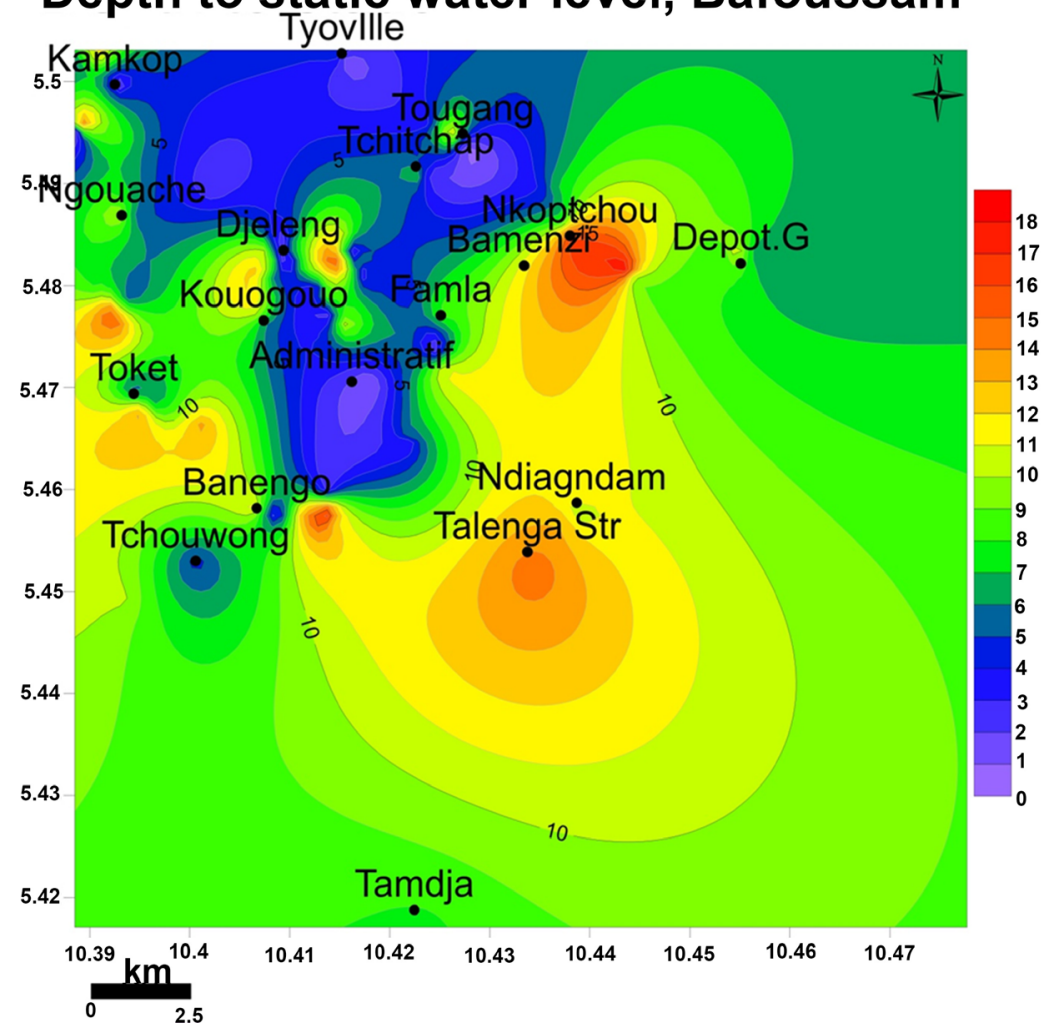

Figure 5. Spatial variation of depth to static water level; note low water levels at Bamenzi, Talenga and Nkoptchou while high value are at Admimistratif, Tchichap Tougang, Tyoville, Tchouwong and Djeleng.

\subsection{4. $\mathrm{pH}$}

The $\mathrm{pH}$ value of most of the groundwater samples in the study area ranged from $4.47-7.84$ (Figure 8). This indicates groundwater in study area is slightly acidic to per-alkaline in nature.

\subsubsection{Electrical Conductivity}

The EC of the groundwater varies from $10-820 \mu \mathrm{S} / \mathrm{cm}$ (Figure 9). These are low values indicating fresh groundwater.

\subsubsection{Total Dissolved Solids}

The total dissolved solids values ranged from 6.7 - $549.4 \mathrm{mg} / \mathrm{L}$ (Figure 10). These are low values indicating fresh groundwater. 


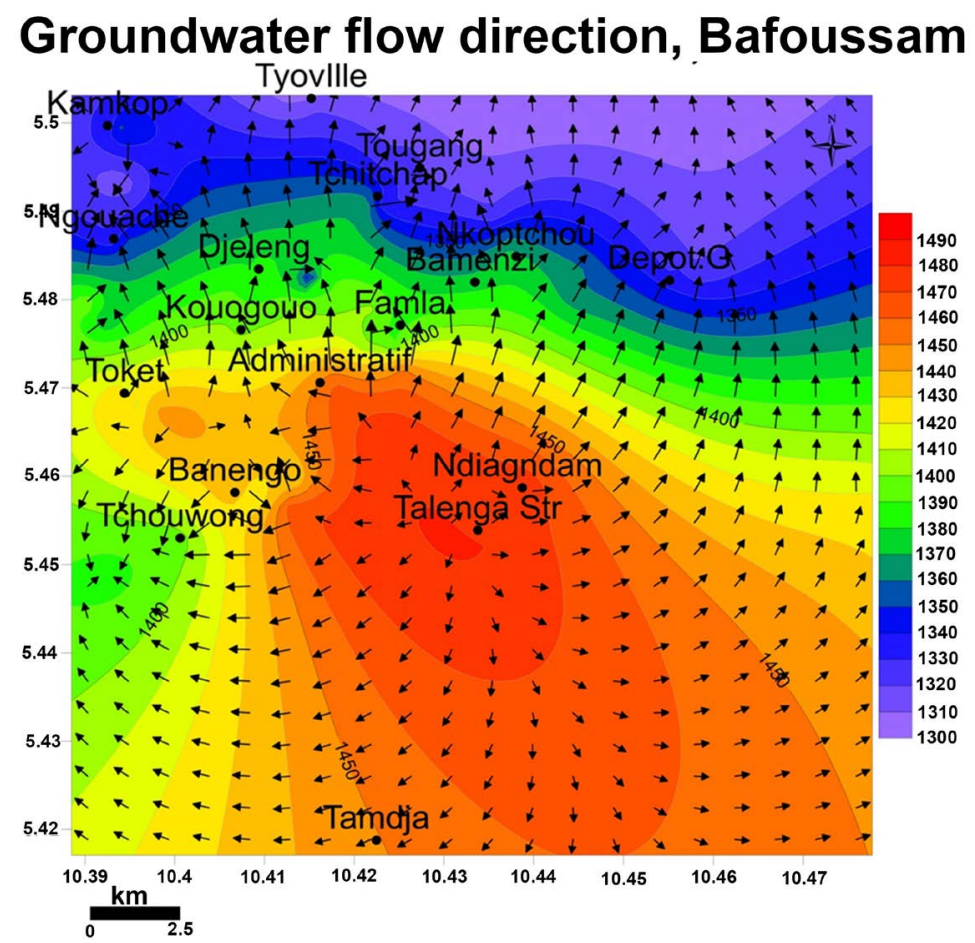

Figure 6. Groundwater flow contours in Bafoussam indicate water flows from the central highest points at Talenga radiating outwards to the surrounding low lying areas; Tchouwong, Tamja, Depot G. and Ngouache in consonance with the topography: Piston flow.

\section{Temperature, Bafoussam}

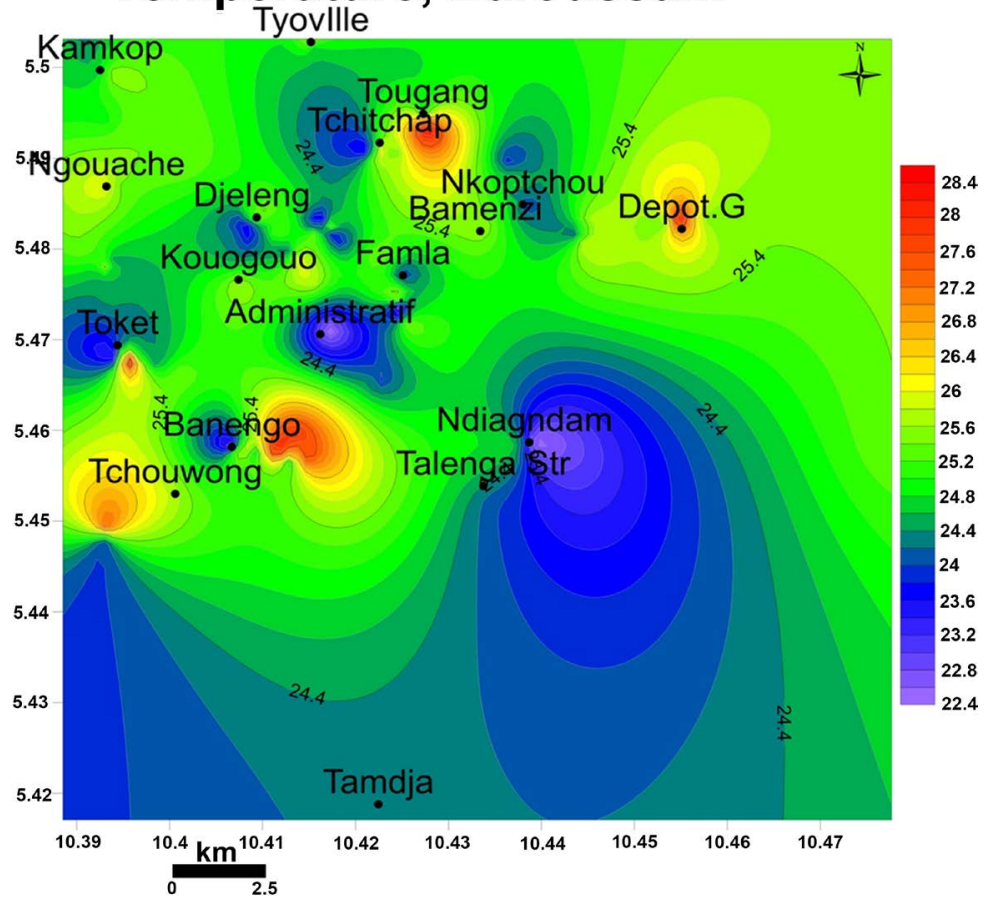

Figure 7. Spatial variation of Bafoussam groundwater temperature with high value at Tougang, Depot $G$ and Tchouwong whereas low values are at Ndiangdam, Toket, Talenga and Administratif. 


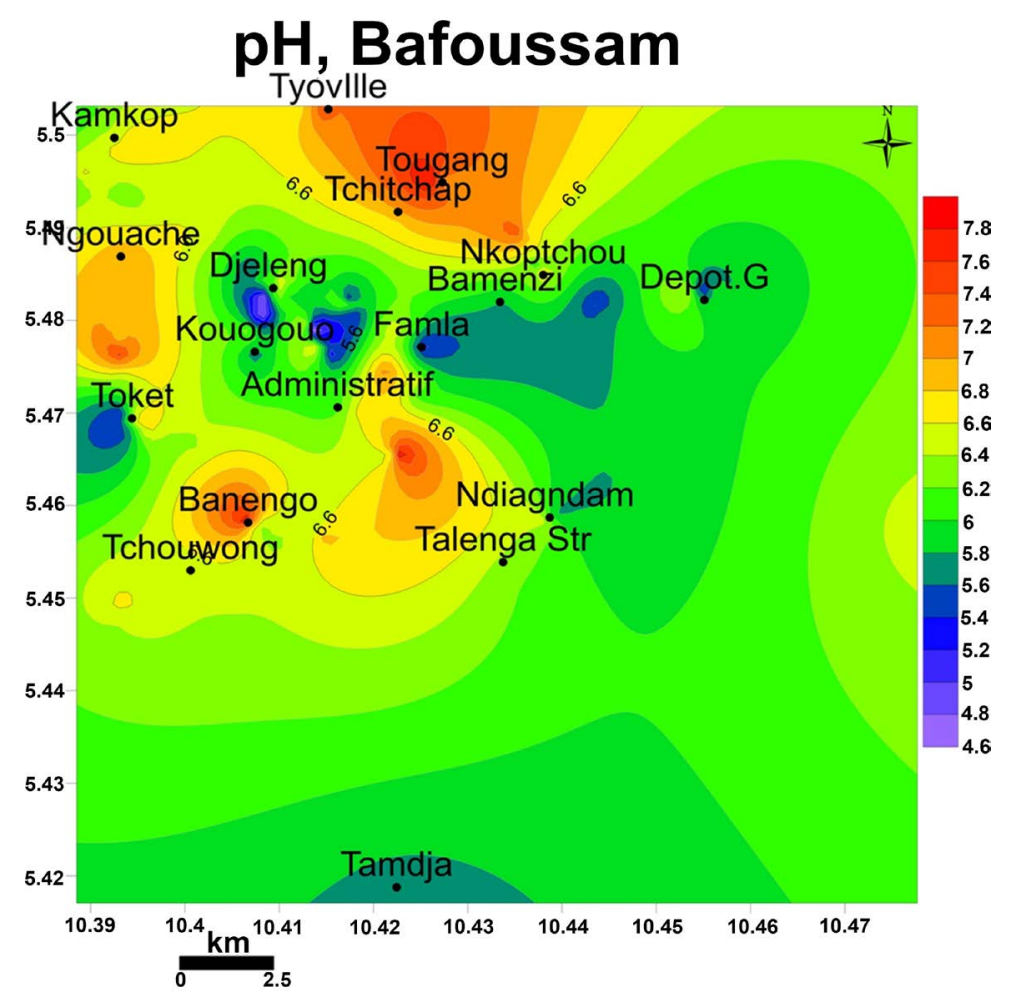

Figure 8. Spatial variation of Bafoussam groundwater $\mathrm{pH}$ with high values at Tougang, Tchitchap, Tyoville and Banengo whereas low values are at Famla, Toket and Djeleng.

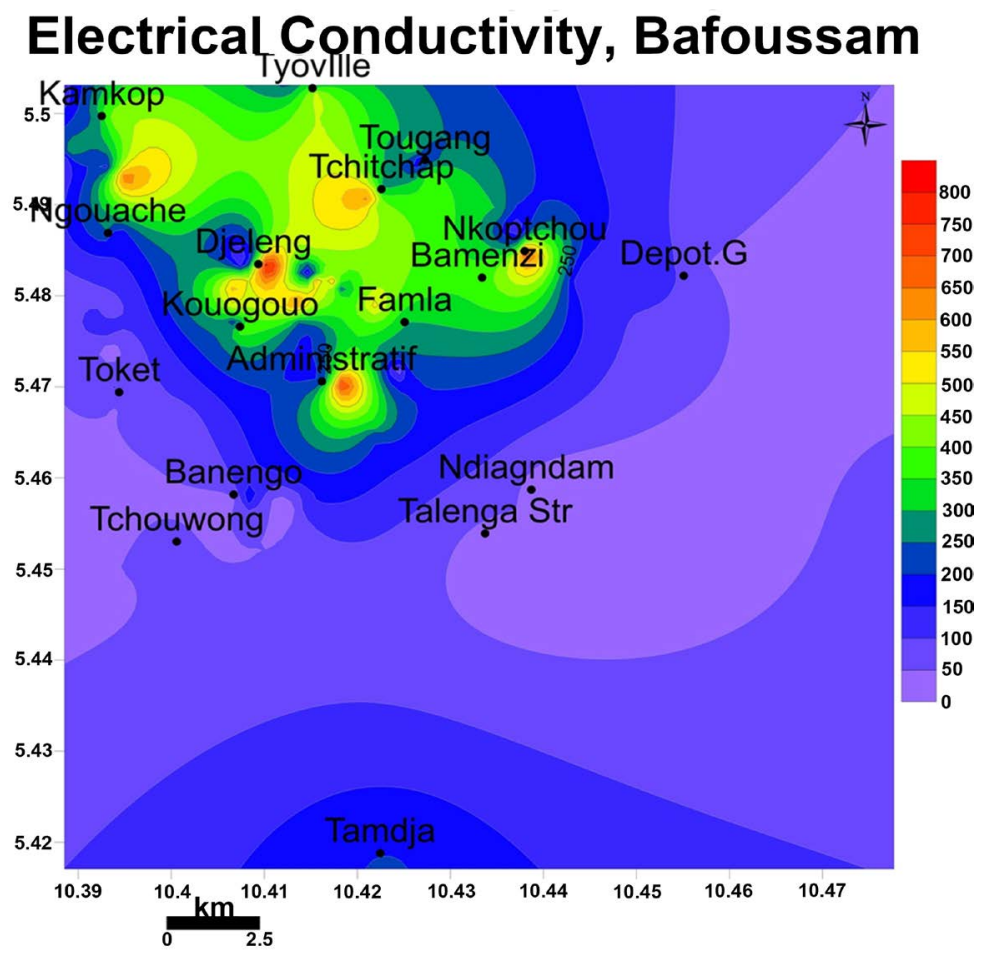

Figure 9. Spatial variation of Bafoussam groundwater EC with high values at Djeleng, Tchitchap, Bamenzi, Administratif and Tyoville whereas low values are in Tamdja and Tougang. 


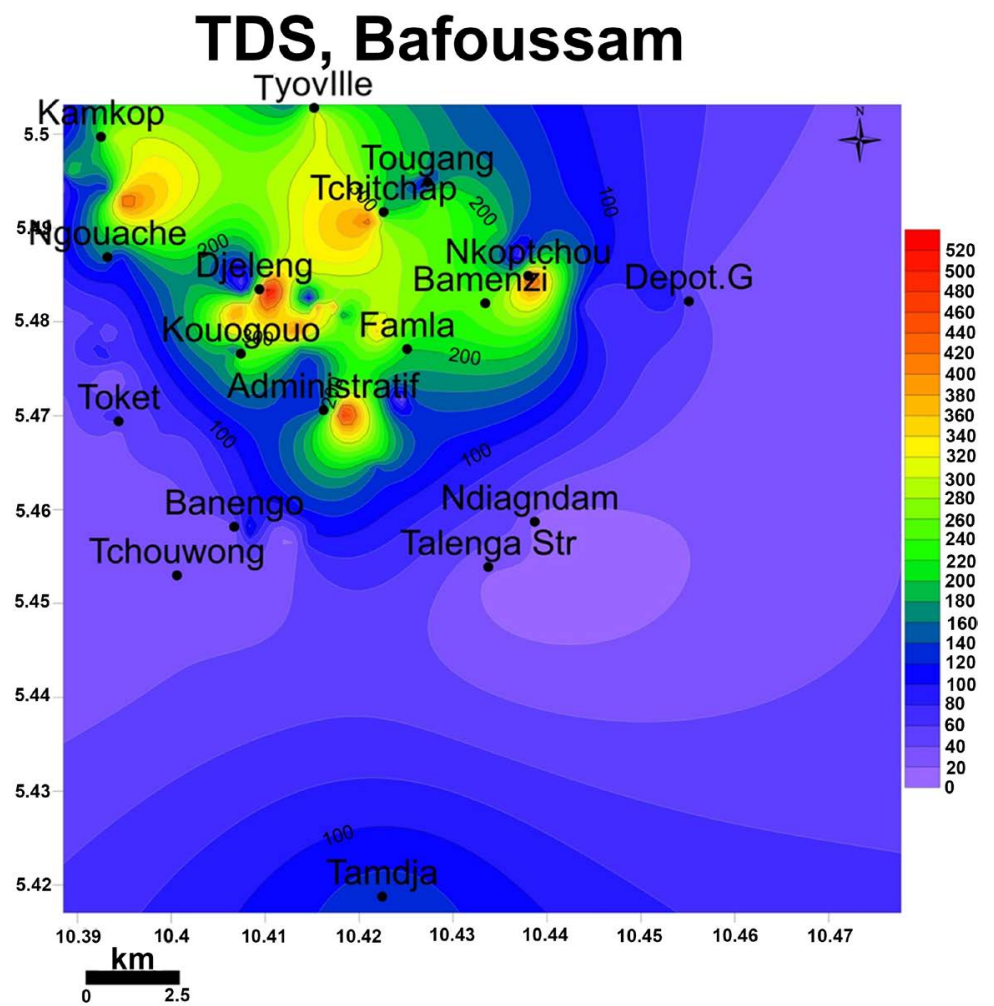

Figure 10. Spatial variation of Bafoussam groundwater TDS with relatively high values at Djeleng, Tchitchap, Bamenzi, Administratif and Tyoville whereas low values are values Tamdja and Tougang.

\subsection{Chemical Parameters of Groundwater}

The results of chemical analysis of major ions; the values of streams, rivers and groundwater are similar indicating connectivity typical of recharge zones in phreatic aquifers (Table 4; Figure 11). The major ions fall below WHO acceptable limits [22]. The sequences of abundance of major ions were, $\mathrm{Ca}^{2+}>\mathrm{Mg}^{2+}>$ $\mathrm{K}^{+}>\mathrm{Na}^{+}>\mathrm{NH}_{4}^{+}, \mathrm{HCO}_{3}^{-}>\mathrm{Cl}>\mathrm{SO}_{4}^{2-}>\mathrm{NO}_{3}>\mathrm{HPO}_{4}^{2-}$. Nitrates and phosphate ions have very low values indicating insignificant pollution and anthropogenic influences except in Famla, Tchouwong and Tougang.

\subsection{Mechanism Controlling Water Chemistry}

\subsubsection{Ionic Ratios of Groundwater in Bafoussam}

18 selected ionic ratios were determined and used to deduce formation inputs in groundwater in Bafoussam (Table 5(a) and Table 5(b)).

12 of the $18(66.7 \%)$ ionic ratios gave indices indicating weathering of geologic formations in Bafoussam as a source of solute concentration in the groundwater while the nitrate-anion ratio indicates there has been no significant anthropogenic contribution to the groundwater chemical content in Bafoussam.

Sulfate indices indicate no oxidation of sulfides and no dedolomitization.

$\mathrm{Ca}$ is sourced from gypsum while $\mathrm{Na}$ is sourced from halite-albite and ion exchange. $\mathrm{Mg}$ is contributed by dolomite dissolution, calcite precipitation or saltwater. There is no plagioclase weathering. 
Table 4. Results of chemical analysis: The values of streams, Rivers and groundwater are similar indicating connectivity typical of recharge zones in phreatic aquifers.

\begin{tabular}{|c|c|c|c|c|c|c|c|c|c|c|c|}
\hline SN & Names (mg/l) & $\mathrm{Na}^{+}$ & $\mathrm{K}^{+}$ & $\mathrm{Ca}^{2+}$ & $\mathrm{Mg}^{2+}$ & $\mathrm{NH}_{4}^{+}$ & $\mathrm{HCO}_{3}$ & $\mathrm{NO}_{3}^{-}$ & $\mathrm{SO}_{4}^{2-}$ & $\mathrm{Cl}^{-}$ & $\mathrm{HPO}_{4}^{2-}$ \\
\hline 1 & FamlaStr & 0.83 & 7.61 & 32.58 & 0.59 & 1.41 & 61.00 & 3.58 & 2.47 & 16.00 & 0.01 \\
\hline 2 & Bamenzi II & 1.38 & 18.41 & 49.96 & 5.04 & 0.00 & 54.90 & 0.00 & 1.89 & 32.00 & 0.03 \\
\hline 3 & Djeleng I & 1.38 & 14.47 & 45.62 & 5.21 & 1.21 & 2.44 & 0.00 & 5.21 & 44.00 & 0.01 \\
\hline 4 & Bamenzi I & 0.85 & 6.44 & 32.58 & 0.74 & 2.02 & 35.38 & 0.00 & 0.00 & 22.00 & 0.04 \\
\hline 5 & Famla & 0.78 & 4.68 & 28.24 & 0.55 & 0.00 & 0.00 & 0.00 & 0.53 & 36.00 & 0.00 \\
\hline 6 & TalengaStr & 0.41 & 2.18 & 26.06 & 0.81 & 0.00 & 28.06 & 0.00 & 2.22 & 0.00 & 0.03 \\
\hline 7 & Tchouwong & 0.44 & 1.60 & 30.42 & 0.58 & 0.54 & 8.54 & 1.64 & 1.40 & 6.00 & 0.00 \\
\hline 8 & Djeleng III & 1.38 & 15.76 & 49.96 & 5.43 & 0.30 & 39.04 & 0.01 & 1.44 & 58.00 & 0.00 \\
\hline 9 & Djeleng IV & 1.86 & 24.8 & 60.82 & 45.6 & 0.53 & 95.16 & 0.78 & 1.33 & 76.00 & 0.00 \\
\hline 10 & Ndiangdam & 0.6 & 3.51 & 32.58 & 10.23 & 0.00 & 0.00 & 0.02 & 0.00 & 2.00 & 0.00 \\
\hline 11 & Djeleng V & 0.92 & 7.88 & 36.92 & 5.87 & 0.00 & 71.98 & 0.00 & 0.14 & 18.00 & 0.03 \\
\hline 12 & Admin & 0.99 & 9.63 & 39.1 & 5.43 & 0.44 & 29.28 & 0.00 & 0.17 & 16.00 & 0.01 \\
\hline 13 & Tchitchap & 0.31 & 4.95 & 32.58 & 10.32 & 0.00 & 54.90 & 0.00 & 2.27 & 14.00 & 0.02 \\
\hline 14 & Kouogouo & 1.54 & 18.53 & 52.12 & 10.59 & 0.00 & 0.00 & 0.00 & 6.34 & 56.00 & 0.00 \\
\hline 15 & Tougang & 0.76 & 3.51 & 32.58 & 10.33 & 1.45 & 29.28 & 1.04 & 2.09 & 24.00 & 0.01 \\
\hline 16 & Tyoville & 1.17 & 12.83 & 45.62 & 5.21 & 0.00 & 102.48 & 0.00 & 1.99 & 10.00 & 0.05 \\
\hline 17 & Nkoptchou & 1.17 & 20.01 & 58.64 & 1.70 & 0.00 & 73.20 & 0.00 & 1.13 & 92.00 & 0.02 \\
\hline 18 & Toket & 0.69 & 3.94 & 30.4 & 0.57 & 0.00 & 39.04 & 0.00 & 0.00 & 4.00 & 0.02 \\
\hline 19 & Depot G. & 0.74 & 2.93 & 32.58 & 9.97 & 0.00 & 0.00 & 0.00 & 0.00 & 4.00 & 0.00 \\
\hline 20 & Ngouache & 0.83 & 4.95 & 36.92 & 8.45 & 0.63 & 37.82 & 0.36 & 0.04 & 0.00 & 0.02 \\
\hline 21 & Tamdja & 0.76 & 3.00 & 30.89 & 0.65 & 1.69 & 70.33 & 0.00 & 0.00 & 4.00 & 0.02 \\
\hline 22 & Djelengi II & 0.4 & 2.16 & 25.99 & 0.71 & 1.59 & 30.98 & 0.01 & 1.20 & 5.00 & 0.03 \\
\hline \multirow[t]{5}{*}{23} & Banengo & 0.31 & 1.80 & 29.99 & 0.60 & 0.00 & 9.21 & 0.00 & 0.37 & 0.00 & 0.00 \\
\hline & Min & 0.31 & 1.60 & 25.99 & 0.55 & 0.00 & 0.00 & 0.00 & 0.00 & 0.00 & 0.00 \\
\hline & $\operatorname{Max}$ & 1.86 & 24.80 & 60.82 & 45.6 & 2.02 & 102.48 & 3.58 & 6.34 & 92.00 & 0.05 \\
\hline & Mean & 0.89 & 8.50 & 37.96 & 6.31 & 0.51 & 37.96 & 0.32 & 1.40 & 23.43 & 0.02 \\
\hline & Std. & 0.42 & 6.93 & 10.31 & 9.40 & 0.68 & 30.90 & 0.82 & 1.64 & 25.82 & 0.01 \\
\hline
\end{tabular}

These high indices values are found in the following localities Ngouache, Toket, Djeleng, Ndiangdam and Tchouwong.

\subsubsection{Dominant Hydrogeochemical Processes in Bafoussam Groundwater}

From the Gibbs diagram, all groundwater samples in the study area plot in the rock-weathering dominance field (Figure 12). 
Table5. (a) Ionic ratios of groundwater in Bafoussam [8]; (b) Interpretation of Ionic ratios with determined formation input [8].

(a)

\begin{tabular}{|c|c|c|c|c|c|c|c|c|c|c|c|c|c|c|c|c|c|c|}
\hline SN & $\begin{array}{c}\mathrm{SO}_{4} / \\
\mathrm{Cl}\end{array}$ & $\begin{array}{c}\mathrm{Na} / \\
\mathrm{Cl}\end{array}$ & $\begin{array}{c}\mathrm{Mg} / \\
\mathrm{Cl}\end{array}$ & $\begin{array}{c}\mathrm{Na} / \\
\mathrm{HCO}_{3}\end{array}$ & $\begin{array}{c}\mathrm{Ca} / \\
\mathrm{HCO}_{3}\end{array}$ & $\begin{array}{l}\mathrm{Ca} / \\
\mathrm{SO}_{4}\end{array}$ & $\begin{array}{l}\mathrm{Ca} / \\
\mathrm{Mg}\end{array}$ & $\begin{array}{c}\mathrm{Ca}+\mathrm{Mg} / \\
\mathrm{Na}+\mathrm{K}\end{array}$ & $\begin{array}{c}\mathrm{HCO}_{3} / \\
\sum \mathrm{An}\end{array}$ & $\begin{array}{l}\mathrm{NO}_{3} / \\
\sum \mathrm{An}\end{array}$ & $\begin{array}{l}\text { SO4/ } \\
\sum A n\end{array}$ & $\begin{array}{c}\mathrm{Mg} / \\
\mathrm{Ca}\end{array}$ & $\begin{array}{c}\mathrm{Na} / \\
\mathrm{Na}+\mathrm{Cl}\end{array}$ & $\begin{array}{c}\mathrm{Mg} / \\
\mathrm{Ca}+\mathrm{Mg}\end{array}$ & $\begin{array}{c}\mathrm{Ca} / \\
\mathrm{Ca}+\mathrm{SO}_{4}\end{array}$ & $\begin{array}{c}\mathrm{Ca}+\mathrm{Mg} / \\
\mathrm{SO}_{4}\end{array}$ & $\begin{array}{c}\mathrm{Cl} / \\
\sum \mathrm{An}\end{array}$ & $\begin{array}{c}\mathrm{Na}+\mathrm{K}-\mathrm{Cl} \\
/ \mathrm{Na}+\mathrm{k}- \\
\mathrm{Cl}-\mathrm{Ca}\end{array}$ \\
\hline 1 & 0.15 & 0.05 & 0.04 & 0.01 & 0.53 & 13.19 & 0.00 & 0.00 & 0.04 & 0.00 & 0.00 & 0.02 & 0.05 & 0.02 & 0.93 & 13.43 & 0.01 & -0.30 \\
\hline 2 & 0.06 & 0.04 & 0.16 & 0.03 & 0.91 & 26.43 & 9.91 & 2.78 & 0.04 & 0.00 & 0.00 & 0.10 & 0.04 & 0.09 & 0.96 & 29.10 & 0.02 & -0.32 \\
\hline 3 & 0.12 & 0.03 & 0.12 & 0.57 & 18.70 & 8.76 & 8.76 & 3.21 & 0.00 & 0.00 & 0.00 & 0.11 & 0.03 & 0.10 & 0.90 & 9.76 & 0.03 & -1.61 \\
\hline 4 & 0.00 & 0.04 & 0.03 & 0.02 & 0.92 & 0.00 & 44.03 & 4.57 & 0.02 & 0.00 & 0.00 & 0.02 & 0.04 & 0.02 & 1.00 & 0.00 & 0.02 & -0.82 \\
\hline 6 & 0.00 & 0.00 & 0.00 & 0.01 & 0.93 & 0.00 & 0.00 & 0.00 & 0.02 & 0.00 & 0.00 & 0.03 & 1.00 & 0.03 & 0.92 & 12.10 & 0.00 & 0.09 \\
\hline 7 & 0.23 & 0.07 & 0.10 & 0.05 & 3.56 & 0.00 & 52.45 & 15.20 & 0.01 & 0.00 & 0.00 & 0.02 & 0.07 & 0.02 & 0.96 & 22.14 & 0.00 & -0.15 \\
\hline 8 & 0.02 & 0.02 & 0.09 & 0.04 & 1.28 & 34.69 & 0.00 & 3.23 & 0.03 & 0.00 & 0.00 & 0.11 & 0.02 & 0.10 & 0.97 & 38.47 & 0.04 & -4.49 \\
\hline 9 & 0.02 & 0.02 & 0.60 & 0.02 & 0.64 & 45.73 & 1.33 & 3.99 & 0.07 & 0.00 & 0.00 & 0.75 & 0.02 & 0.43 & 0.98 & 80.02 & 0.05 & -4.30 \\
\hline 10 & 0.00 & 0.30 & 5.12 & 0.00 & 0.00 & 0.00 & 0.00 & 0.00 & 0.00 & 0.00 & 0.00 & 0.31 & 0.23 & 0.24 & 1.00 & 0.00 & 0.00 & 0.06 \\
\hline 11 & 0.01 & 0.05 & 0.33 & 0.01 & 0.51 & 0.00 & 6.29 & 4.86 & 0.05 & 0.00 & 0.00 & 0.16 & 0.05 & 0.14 & 1.00 & 305.64 & 0.01 & -0.33 \\
\hline 13 & 0.16 & 0.02 & 0.74 & 0.01 & 0.59 & 14.35 & 3.16 & 0.00 & 0.04 & 0.00 & 0.00 & 0.32 & 0.02 & 0.24 & 0.93 & 18.90 & 0.01 & -0.37 \\
\hline 14 & 0.11 & 0.03 & 0.19 & 0.00 & 0.00 & 0.00 & 4.92 & 0.00 & 0.00 & 0.00 & 0.00 & 0.20 & 0.03 & 0.17 & 0.89 & 9.89 & 0.04 & -2.22 \\
\hline 15 & 0.09 & 0.03 & 0.43 & 0.03 & 1.11 & 15.59 & 3.15 & 10.05 & 0.02 & 0.00 & 0.00 & 0.32 & 0.03 & 0.24 & 0.94 & 20.53 & 0.02 & -1.54 \\
\hline 16 & 0.20 & 0.12 & 0.52 & 0.01 & 0.45 & 22.92 & 8.76 & 3.63 & 0.07 & 0.00 & 0.00 & 0.11 & 0.10 & 0.10 & 0.96 & 25.54 & 0.01 & 0.08 \\
\hline 17 & 0.01 & 0.01 & 0.02 & 0.00 & 0.00 & 0.00 & 34.49 & 0.00 & 0.05 & 0.00 & 0.00 & 0.03 & 0.01 & 0.03 & 0.98 & 53.40 & 0.06 & 5.81 \\
\hline 18 & 0.00 & 0.17 & 0.14 & 0.02 & 0.78 & 0.00 & 53.33 & 6.69 & 0.03 & 0.00 & 0.00 & 0.02 & 0.15 & 0.02 & 1.00 & 0.00 & 0.00 & 0.02 \\
\hline 19 & 0.00 & 0.19 & 2.49 & 0.00 & 0.00 & 0.00 & 3.27 & 11.59 & 0.00 & 0.00 & 0.00 & 0.31 & 0.16 & 0.23 & 1.00 & 0.00 & 0.00 & -0.01 \\
\hline 20 & 0.00 & 0.00 & 0.00 & 0.02 & 0.98 & 923.00 & 4.37 & 7.85 & 0.03 & 0.00 & 0.00 & 0.23 & 1.00 & 0.19 & 1.00 & 1134.25 & 0.00 & 0.14 \\
\hline 21 & 0.00 & 0.19 & 0.16 & 0.01 & 0.44 & 0.00 & 47.52 & 8.39 & 0.05 & 0.00 & 0.00 & 0.02 & 0.16 & 0.02 & 1.00 & 0.00 & 0.00 & -0.01 \\
\hline 22 & 0.24 & 0.08 & 0.14 & 0.01 & 0.84 & 21.66 & 36.61 & 10.43 & 0.02 & 0.00 & 0.00 & 0.03 & 0.07 & 0.03 & 0.96 & 22.25 & 0.00 & -0.10 \\
\hline 23 & 0.00 & 0.00 & 0.00 & 0.03 & 3.26 & 81.05 & 49.98 & 14.50 & 0.01 & 0.00 & 0.00 & 0.02 & 1.00 & 0.02 & 0.99 & 82.68 & 0.00 & 0.07 \\
\hline Min & 0.00 & 0.00 & 0.00 & 0.00 & 0.00 & 0.00 & 0.00 & 0.00 & 0.00 & 0.00 & 0.00 & 0.02 & 0.01 & 0.02 & 0.89 & 0.00 & 0.00 & -4.49 \\
\hline Std. & 0.08 & 0.08 & 1.13 & 0.12 & 3.83 & 190.45 & 21.11 & 4.67 & 0.02 & 0.00 & 0.00 & 0.17 & 0.33 & 0.11 & 0.03 & 239.65 & 0.02 & 3.51 \\
\hline
\end{tabular}

(b)

\begin{tabular}{|c|c|c|c|}
\hline Ionic ratio & Range & Comment & Interpretation \\
\hline $\mathrm{SO}_{4} / \mathrm{Cl}$ & $0.00-0.24$ & High & Additional sources of $\mathrm{SO}_{4}$ from weathering of sulfates \\
\hline $\mathrm{Na} / \mathrm{Cl}$ & $0.00-0.30$ & Low & No Na-adsorption during freshening and a little silicate weathering \\
\hline $\mathrm{Mg} / \mathrm{Cl}$ & $0.00-5.12$ & High & Cation-exchange and silicate weathering of sandstones \\
\hline $\mathrm{Na} / \mathrm{HCO}_{3}$ & $0.00-0.57$ & Very low & No Substantial weathering of Na-feldspar or other Na-silicates \\
\hline $\mathrm{Ca} / \mathrm{HCO}_{3}$ & $0.00-18.7$ & High & Calc-carbonate dissolution or Calc-silicate weathering \\
\hline $\mathrm{Ca} / \mathrm{Mg}$ & $0.00-53.3$ & High & Cation-exchange and weathering of silicate rocks \\
\hline $\mathrm{Ca}+\mathrm{Mg} / \mathrm{Na}+\mathrm{K}$ & $0.00-15.20$ & High & Carbonate weathering \\
\hline $\mathrm{HCO}_{3} / \Sigma$ Anions & $0.00-0.07$ & High & Weathering reactions and input of dissolved species in recharge area \\
\hline $\mathrm{NO}_{3} / \sum$ Anions & $0.00-2.5 \mathrm{E}-3$ & Very low & No anthropogenic contribution \\
\hline $\mathrm{SO}_{4} / \Sigma$ Anions & $0.00-4.4 \mathrm{E}-3$ & Very low & No oxidation of sulphides \\
\hline $\mathrm{Mg} / \mathrm{Ca}$ & $0.02-0.75$ & Low & Weathering of Silicate rocks \\
\hline $\mathrm{Mg} / \mathrm{Ca}+\mathrm{Mg}$ & $0.02-0.43$ & High & Dolomite dissolution, calcite precipitation \\
\hline $\mathrm{Ca} / \mathrm{Ca}+\mathrm{SO}_{4}$ & $0.89-1.00$ & High & Calcium source other than gypsum \\
\hline $\mathrm{Ca}+\mathrm{Mg} / \mathrm{SO}_{4}$ & $2.96-1134.25$ & High & No dedolomitization \\
\hline $\mathrm{Na}+\mathrm{K}-\mathrm{Cl} / \mathrm{Na}+\mathrm{K}-\mathrm{Cl}+\mathrm{Ca}$ & $-4.49-13.28$ & High & Plagioclase weathering unlikely \\
\hline $\mathrm{Cl} /$ /Anions & $0.00-0.06$ & Low & Rock weathering \\
\hline
\end{tabular}



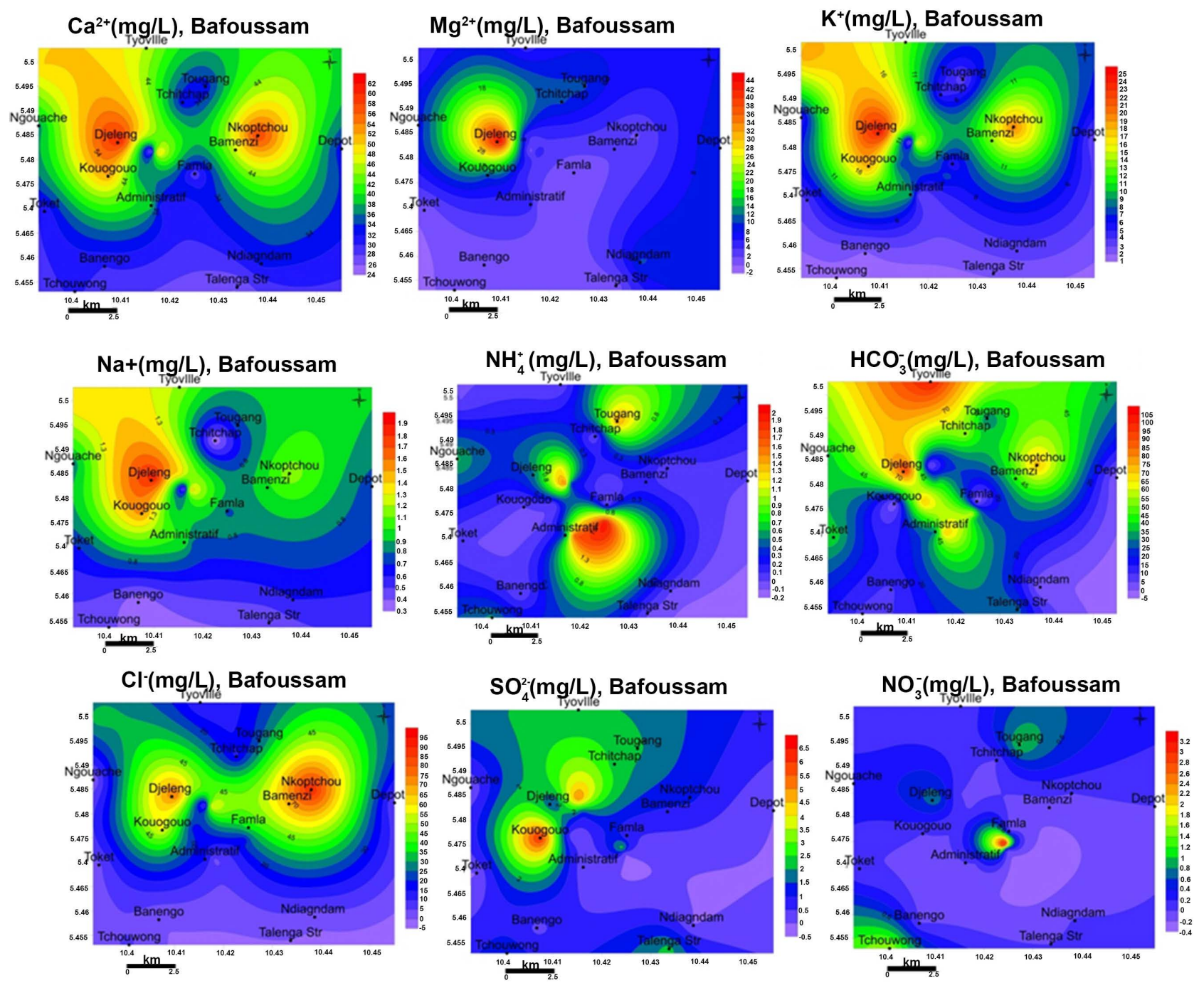

\section{$\mathrm{HPO}_{4}^{2}(\mathrm{mg} / \mathrm{L})$, Bafoussam}

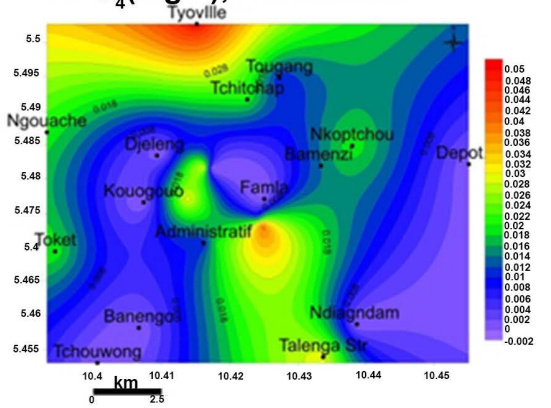

Figure 11. Spatial variations of ionic concentrations of major cations and anions in Bafoussam groundwater: The distribution of the major ionic concentrations reflects the underlying geology of the area.

\subsubsection{Groundwater Types}

The diamond field of piper diagram is divided into seven fields classifying water types and designated with alphabets from A to G [9] [22]. Using this classification, the water from the study area is distinguished into the $\mathrm{A}, \mathrm{B}$, and $\mathrm{C}$ categories. The D, E, F, and G water types are absent. Category A, 8 samples 34.78\%; characterized by normal earth alkaline water with prevailing bicarbonate. 


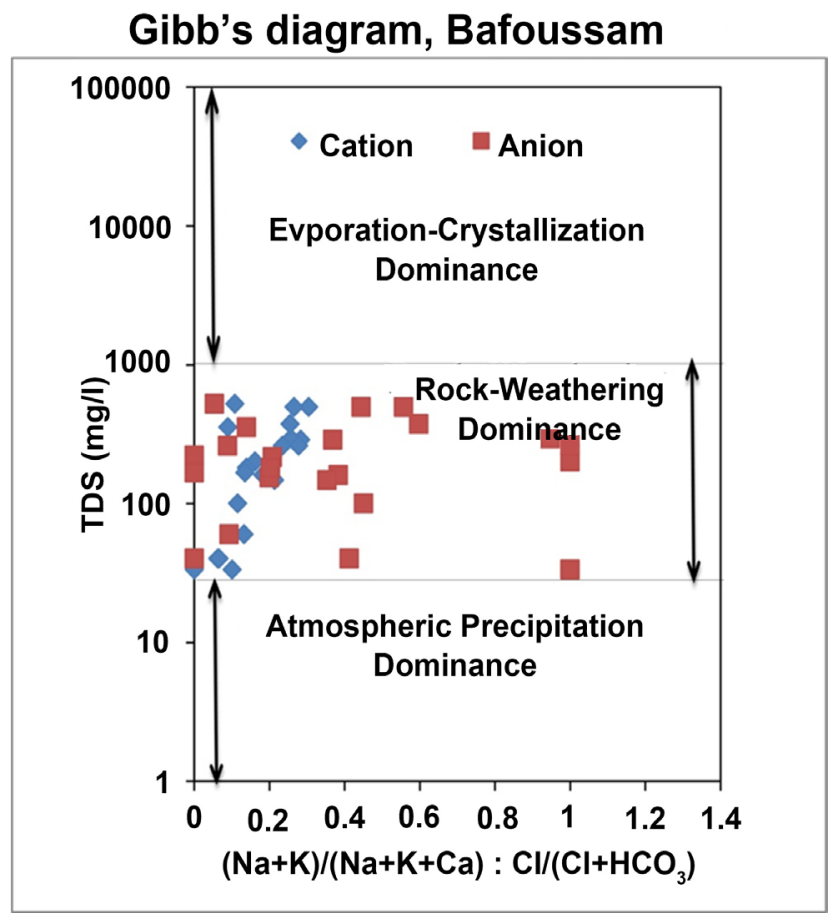

Figure 12. Gibbs diagram indicating the mechanism contributing groundwater constituents in the study area is rock-weathering [9].

Category B, 6 samples $26.09 \%$ are characterized by normal earth alkaline water with prevailing sulfate or chloride and Category C, 9 samples $39.13 \%$ are characterized by alkaline earth water with increased portions of alkalis with prevailing bicarbonate (Table 6). The water types of Bafoussam groundwater are $\mathrm{Ca}-\mathrm{Cl}$ and $\mathrm{Ca}-\mathrm{HCO}_{3}$ (Table 6, Figure 13).

\subsubsection{Hydrogeochemical Facies}

From the Piper diagram, field (I): $\mathrm{Ca}-\mathrm{Mg}-\mathrm{Cl}-\mathrm{SO}_{4}$ hydrogeochemical facies has 14 samples $60.87 \%$; This facies is characteristic of groundwater at some distance along its flow path [23]. Ca- $\mathrm{Mg}-\mathrm{HCO}_{3}$ hydrogeochemical facies field (IV) has 9 samples $39.13 \%$ (Figure 13). This facies is characteristic of freshly recharged groundwater that has equilibrated with $\mathrm{CO}_{2}$ and soluble carbonate minerals under an open system conditions in the vadose zone typical of shallow groundwater flow systems in crystalline phreatic aquifers. No samples plotted on field (II) and field (III) (Table 7).

\subsubsection{Durov Diagram}

Based on the Lloyd and Heathcoat classification [13]: Four classes of processes occur in Bafoussam groundwater; Class 1 recharging waters: 7 samples 30.43\%; Class 2 ion exchange water: 9 samples 39.13\%: Class 3 ion exchange water: 6 samples $26.10 \%$ and Class 5 simple dissolution or mixing: 1 sample $4.34 \%$ respectively (Figure 14). There are no Classes 4, 6, 7, and 9 of groundwater from Bafoussam (Table 8). 
Table 6. Classification of Bafoussam groundwater based on Piper diagram [9] to depict water types.

\begin{tabular}{|c|c|c|c|}
\hline Class & Description of Water Types & No & $\%$ \\
\hline A & Normal earth alkaline water with prevailing bicarbonate & 8 & 34.78 \\
\hline B & $\begin{array}{l}\text { Normal earth alkaline water with prevailing bicarbonate and sulfate or } \\
\text { chloride }\end{array}$ & 6 & 26.09 \\
\hline \multirow[t]{2}{*}{$\mathrm{C}$} & Normal earth alkaline water with prevailing Sulfate or Chloride & 9 & 39.13 \\
\hline & Cations field & & \\
\hline 1 & Calcium rich & 22 & 95.65 \\
\hline \multirow[t]{2}{*}{2} & Magnesium rich & 1 & 4.35 \\
\hline & Anion Field & & \\
\hline 4 & Bicarbonate rich & 11 & 47.83 \\
\hline 5 & Chloride rich & 12 & 52.17 \\
\hline
\end{tabular}

Table 7. Classification of water based on Piper diagram [11].

\begin{tabular}{cccc}
\hline Field & Hydrogeochemical facies & No & $\%$ \\
\hline Field I & Ca-Mg-Cl-SO & 14 & 60.87 \\
Field IV & Ca-Mg- $-\mathrm{HCO}_{3}$ & 9 & 39.13 \\
\hline
\end{tabular}

Table 8. Classification of water based on Durov diagram [13].

\begin{tabular}{|c|c|c|c|}
\hline $\mathrm{SN}$ & Description of Water Types & No & $\%$ \\
\hline 1 & $\begin{array}{l}\mathrm{HCO}_{3} \text { and } \mathrm{Ca} \text { dominant, indicates recharging waters in the Granitoids and } \\
\text { Basalts }\end{array}$ & 7 & 30.43 \\
\hline 2 & This water type is dominated by $\mathrm{Ca}$ and $\mathrm{HCO}_{3}$ ions & 9 & 39.13 \\
\hline 3 & $\begin{array}{l}\mathrm{HCO}_{3} \text { and } \mathrm{Na} \text { are dominant, normally indicates ion exchanged water, although } \\
\text { the generation of } \mathrm{CO}_{2} \text { at depth can produce } \mathrm{HCO}_{3} \text { where } \mathrm{Na} \text { is dominant under } \\
\text { certain circumstances }\end{array}$ & 6 & 26.10 \\
\hline 5 & $\begin{array}{l}\text { No dominant anion or cation, indicates water exhibiting simple dissolution or } \\
\text { mixing }\end{array}$ & 1 & 4.34 \\
\hline
\end{tabular}

\subsection{Water Quality}

\subsubsection{Domestic Water Quality}

Ionic content of water in the study area was used to evaluate groundwater suitability for domestic use: The recommended values are of the WHO guidelines [22].

\subsubsection{Total Hardness}

The total hardness of groundwater samples ranged from $67.88-339.01 \mathrm{mg} / \mathrm{L}$ $\mathrm{CaCO}_{3}$ (Figure 15). 13.04\% of groundwater in the study area can be classified as soft, $73.92 \%$ fell into the moderately hard category and $13.04 \%$ is hard water that may be a potential health risk factor (Table 9). The WHO guideline value of hardness for domestic use is $600 \mathrm{mg} / 1 \mathrm{CaCO}_{3}$, implying that the water suitable for drinking. 

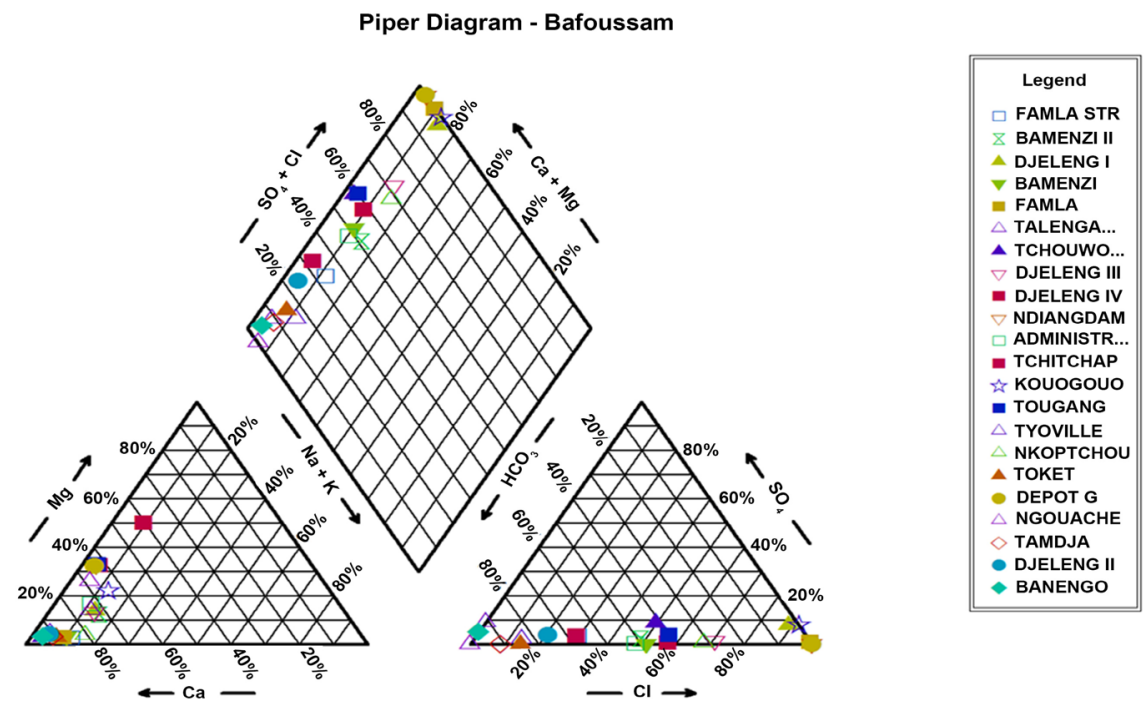

Figure 13. Piper diagram for Bafoussam groundwater samples; Category A, 8 samples $34.78 \%$; characterized by normal earth alkaline water with prevailing bicarbonate; Category B, 6 samples $26.09 \%$ are characterized by normal earth alkaline water with prevailing sulfate or chloride and Category C, 9 samples 39.13\% are characterized by alkaline earth water with increased portions of alkalis with prevailing bicarbonate. Diamond field (I): $\mathrm{Ca}-\mathrm{Mg}-\mathrm{Cl}-\mathrm{SO}_{4}$ hydrogeochemical facies has 14 samples (60.87\%). Field (IV), Ca-Mg- $\mathrm{HCO}_{3}$ hydrogeochemical facies has 9 samples $39.13 \%$. No samples plotted on field (II) and field (III) [10].

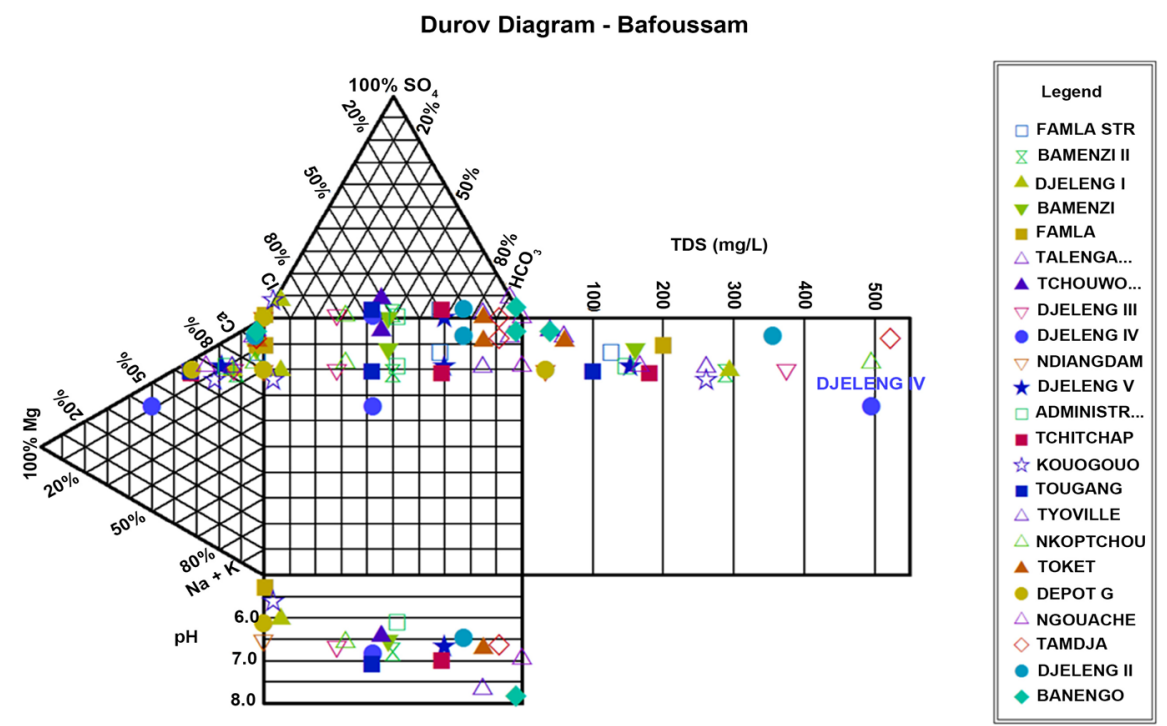

Figure 14. Expanded Durov plot for Bafoussam; Four classes of processes occur in Bafoussam groundwater; Class 1 recharging waters: 7 samples 30.43\%; Class 2 ion exchange water: 9 samples 39.13\%: Class 3 ion exchange water: 6 samples $(26.10 \%)$ and Class 5 simple dissolution or mixing: 1 sample $4.34 \%$ respectively [12] [13].

\subsubsection{Water Quality Index (WQI)}

Water Quality Index (WQI) considered the most effective tool to convey the water quality information in the simplest form to the public [25] The WQI values ranged from 0 - 42.09 (Figure 16). All groundwater samples in the study area 


\section{Water hardness, Bafoussam}

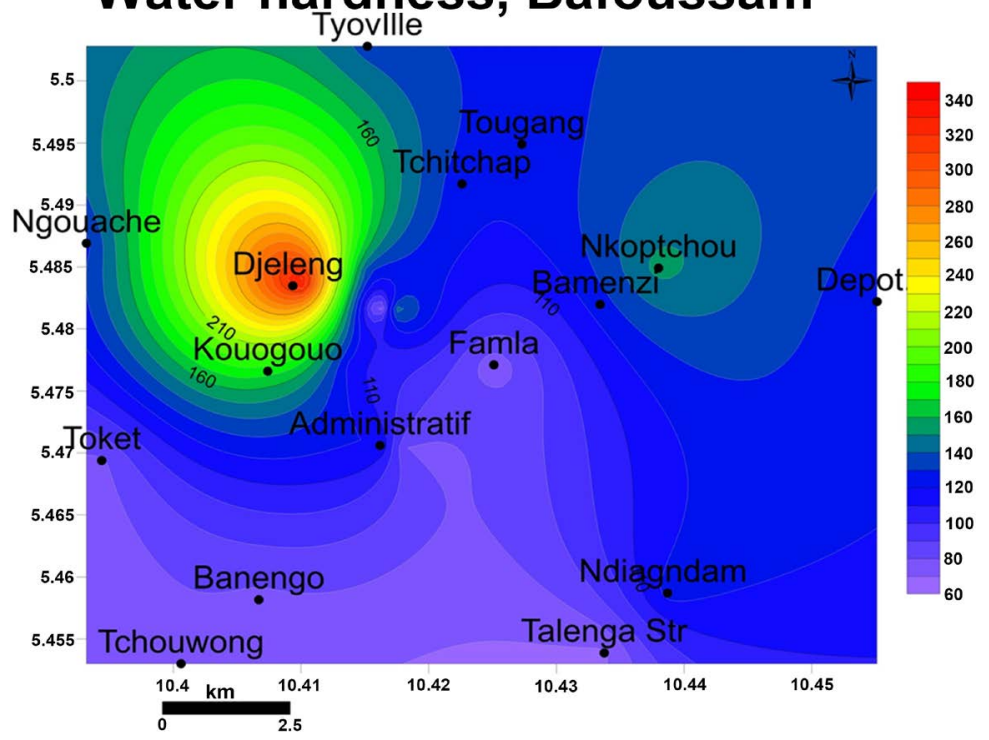

Figure 15. Spatial variation of total hardness with high values at Djeleng and Nkoptchou whereas low values are at Banengo, Toket, Tamdja, Toket, Tchouwong and Famla.

\section{WQI, Bafoussam}

Tyovllle

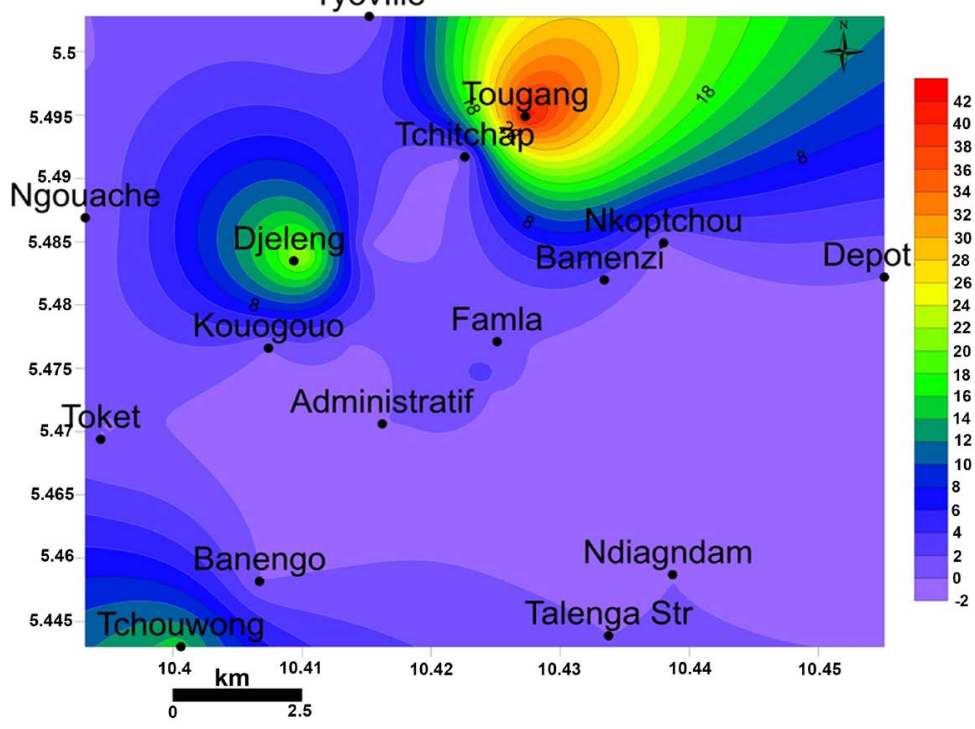

Figure 16. Spatial variation of water quality index (WQI); high values are at Tougang whereas low values are at Administratif, Ndiangndam and Banengo.

Table 9. Hardness classification of groundwater of the study area [24].

\begin{tabular}{cccc}
\hline Hardness $\mathrm{CaCO}_{3}(\mathrm{mg} / \mathrm{L})$ & Water Classification & Number Of Samples & $\%$ \\
\hline $0-75$ & Soft & 3 & 13.04 \\
$76-150$ & Moderately Hard & 17 & 73.92 \\
$151-300$ & Hard & 3 & 13.04 \\
\hline
\end{tabular}


are suitable for domestic and other utilitarian purposes as they belong to excellent to good water quality classes (Table 10).

\subsubsection{Water Quality for Irrigational Use}

The important parameters which determine the irrigation water quality of the study area are discussed below.

\subsubsection{Percent Sodium}

The $\% \mathrm{Na}$ values ranged from 3.69 - 15.50. The quality classifications of irrigation water based on the values of sodium percentage indicate groundwater in the study area fall in the excellent-good and good-permissible category indicates the water is suitable for irrigation [14] (Figure 17).

The values of \% Na vary across Bafoussam with relatively high values at Tyoville, Bamenzi, Nkoptchou and Kouogouo whereas low values are at Banengo and Tchouwong (Figure 18).

\subsubsection{Sodium Adsorption Ratio SAR and EC}

The degree to which the irrigation water tends to enter into cation exchange reactions in the soil can be indicated by the sodium adsorption ratio [26]. SAR values ranged from $0.01-0.05$ (Figure 19). Based on these SAR values and the alkalinity the USSL Salinity diagram for Bafoussam groundwater was plotted (Figure 20). All the 23 groundwater samples fell in the $\mathrm{S}_{1}-\mathrm{C}_{0-3}$ classes of very-low to low sodium hazard thus all the water sources are suitable for irrigation. The groundwater was also classified by the EC values (Table 11), and the all samples were good to excellent.

\subsubsection{Permeability Index}

The classification of irrigation waters has been attempted on the basis of permeability Index [20]. The PI values ranged from 1.05 - 67.98 (Figure 21). The groundwater samples of the study area fell in class-I and II. The groundwater samples of the study area are of good quality for irrigation (Figure 22).

\subsubsection{Magnesium Adsorption Ratio}

Magnesium adsorption ratio values ranged from 2.89 - 55.28 (Figure 23). Magnesium Ratio adsorption less than $50 \%$ it is considered as suitable for irrigation purpose. In the study area, almost all the groundwater samples are suitable for irrigation but for Djeleng.

\subsubsection{Residual Sodium Carbonate}

The RSC values ranged from -5.23 to -0.44 (Figure 24). RSC values $<1.25 \mathrm{mg} / \mathrm{l}$ are considered as safe for irrigation while those from $1.25 \mathrm{mg} / \mathrm{L}$ to $2.5 \mathrm{mg} / \mathrm{L}$ are

Table 10. WQI classification indicating the suitability of water for drinking.

\begin{tabular}{cccc}
\hline Index & Quality & No of samples & $\%$ \\
\hline $0-25$ & Excellent & 22 & 95.6 \\
$26-50$ & Good & 1 & 4.4 \\
\hline
\end{tabular}




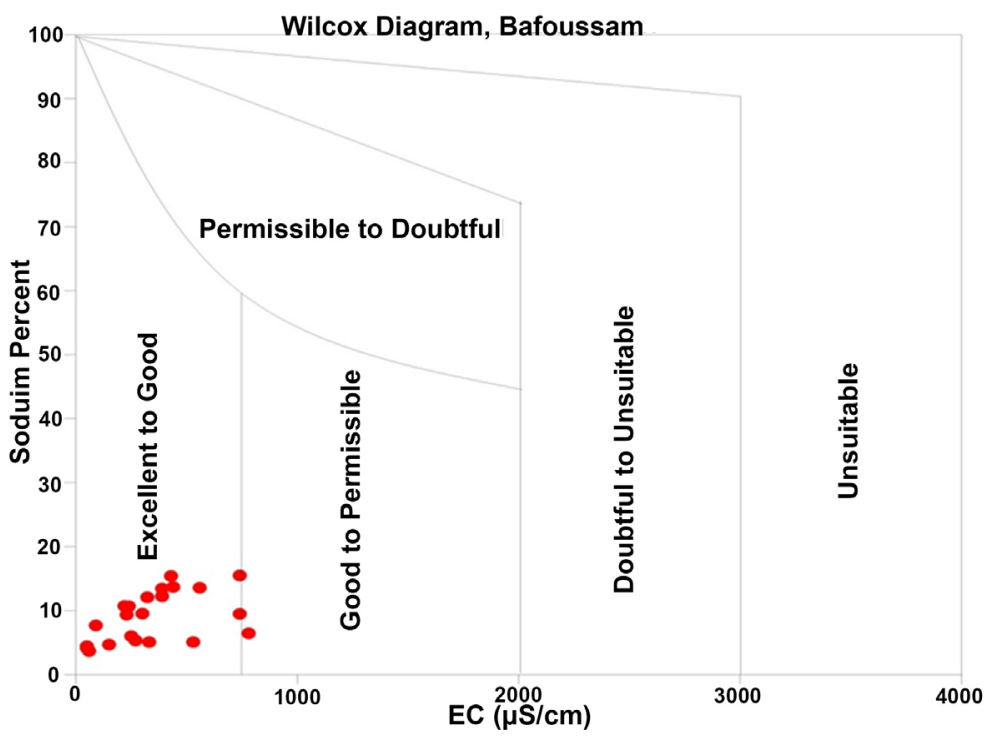

Figure 17. Wilcox diagram of groundwater suitability for irrigation; 19 samples $95 \%$ plot in excellent-good fields and 1 sample 5\% plotting in good-permissible field indicating the water is suitable for irrigation.

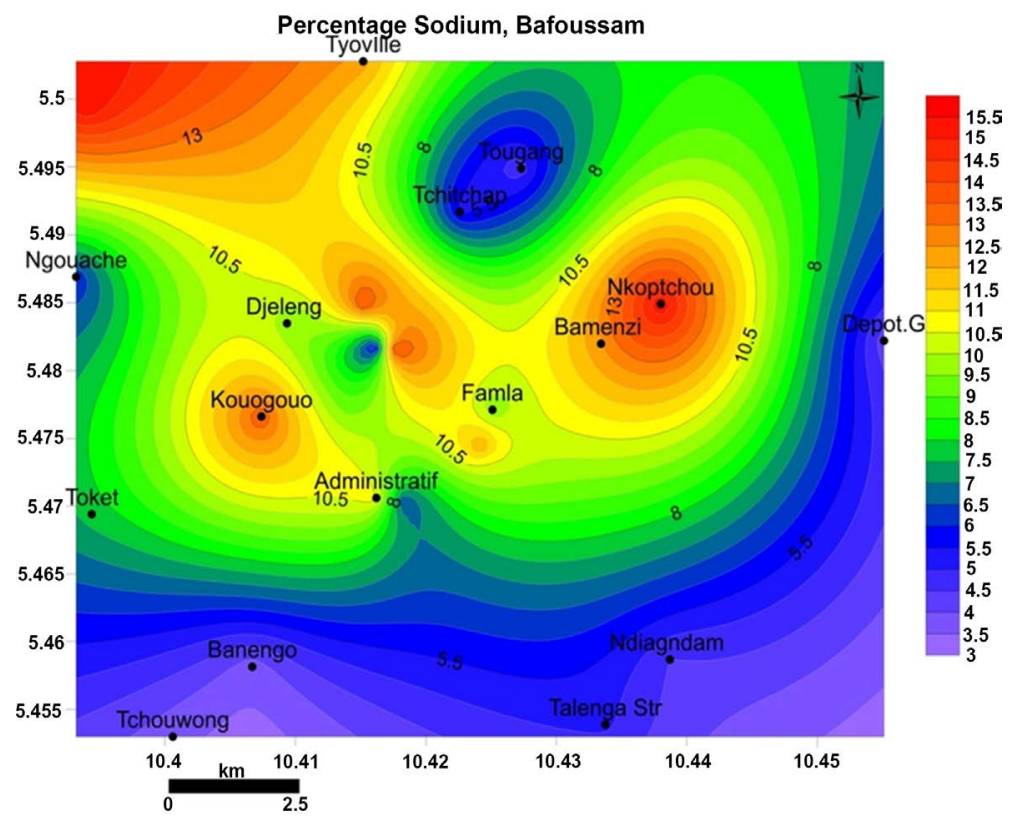

Figure 18. Spatial variation of percent sodium $(\% \mathrm{Na})$ with high values at Tyoville, Bamenzi, Nkoptchou and Kouogouo whereas low values are at Banengo and Tchouwong.

marginally suitable for irrigation. RSC values $>2.5$ the groundwater is unsuitable for irrigation [18] [19]. All the RSC values are $<1.25$ in the study area (Figure 23 ), thus the groundwater in Bafoussam is suitable for irrigation purposes.

\subsubsection{Kelly's Ratio}

$\mathrm{KR}<1$ is considered suitable for irrigation and $\mathrm{KR}>1$ is unsuitable. $\mathrm{KR}$ values vary between $0.005-0.023$. Thus all groundwater samples are suitable for 


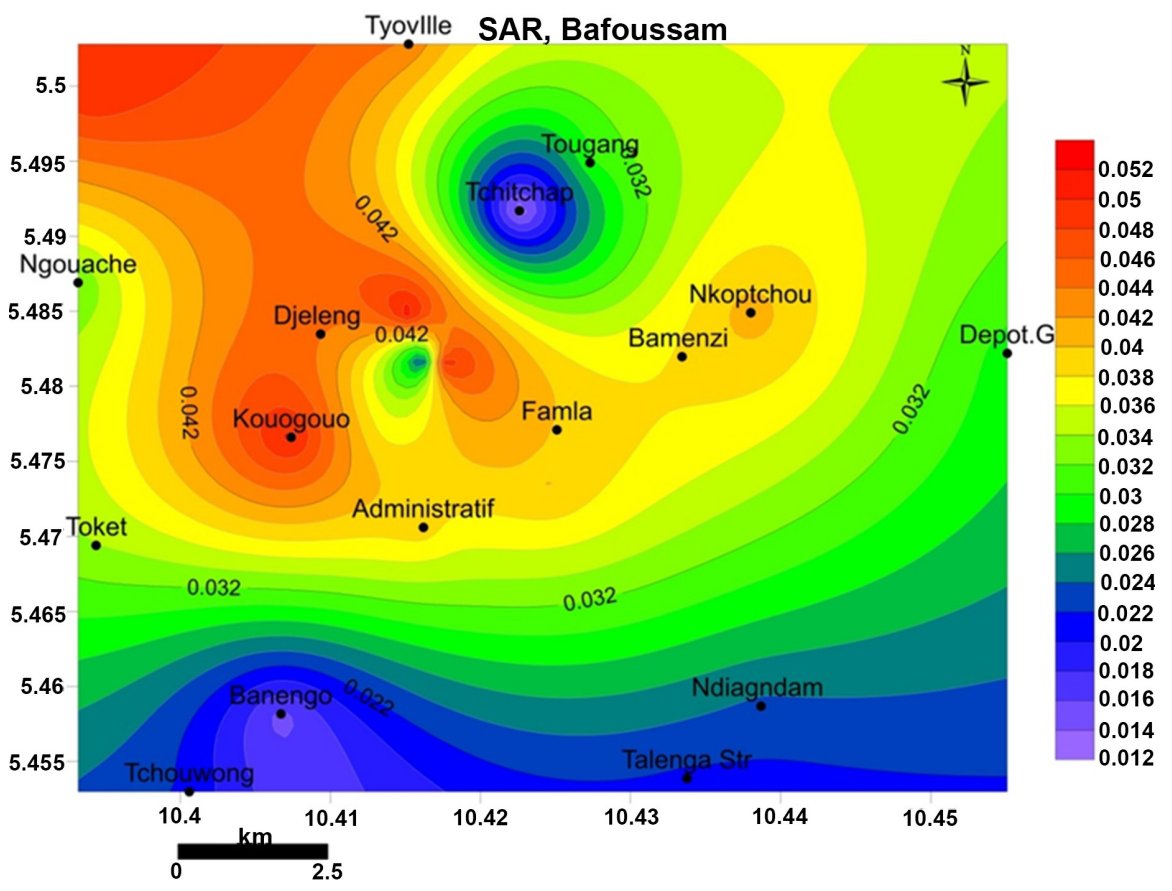

Figure 19. Spatial variation of sodium adsorption ratio with high values at Tyoville, Djeleng and Kouogouo whereas low values are at Banengo and Tchouwong.

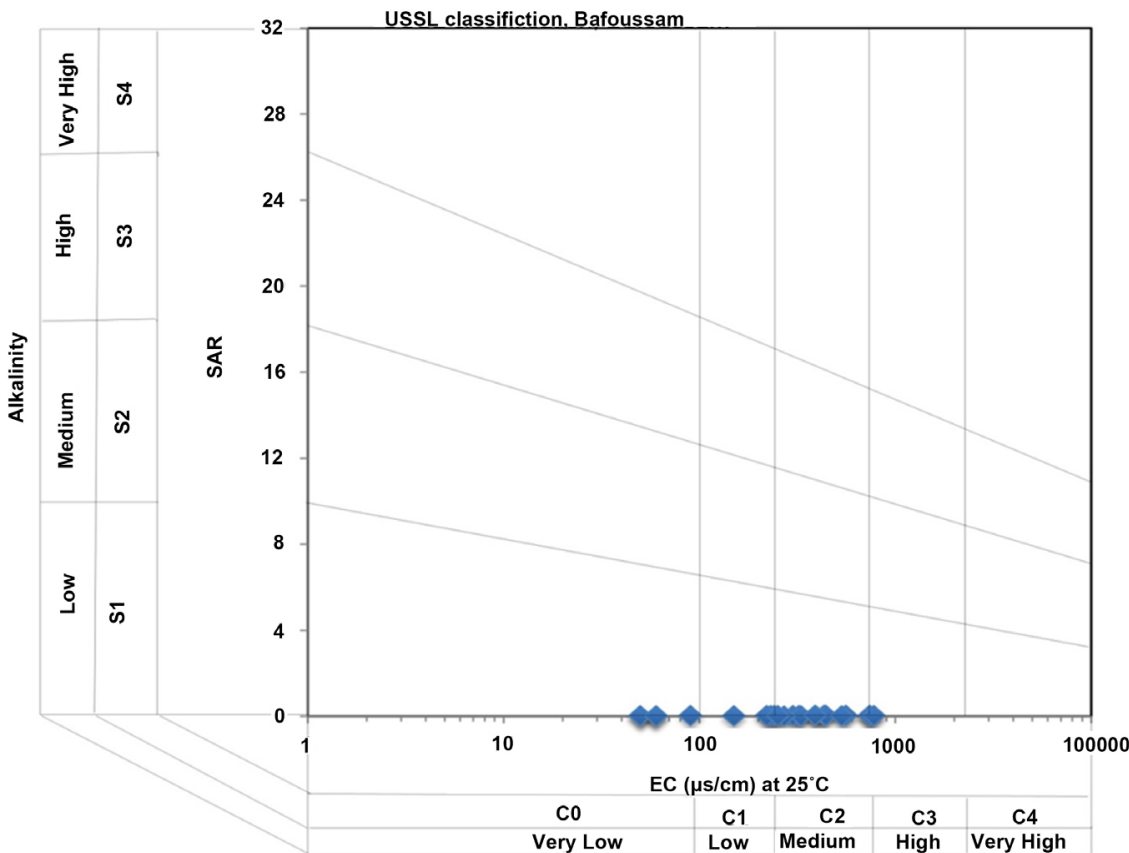

Figure 20. Residual Salinity Hazard classification, Bafoussam; The $S_{1} C_{0}, S_{1} C_{1}$, and $S_{1} C_{2}$ make up the excellent, very good and good classes respectively. 5 samples $21.74 \%$ plotted in the excellent class, 5 samples $21.74 \%$ plotted in the very good class and 13 samples $56.52 \%$ potted in the good class.

irrigation based on Kelly's ratio [15]. Kelly ratios vary across Bafoussam with relatively high values at Famla and Administratif whereas low values are at Banengo and Tougang (Figure 25). 
Table 11. Water quality classification based on EC of Bafoussam groundwater.

\begin{tabular}{ccccc}
\hline Hazard Class & EC $(\mu \mathrm{S} / \mathrm{cm})$ & Quality & No of Samples & $\%$ \\
\hline C0 & $0-100$ & Excellent & 5 & 21.74 \\
C1 & $101-250$ & Very Good & 5 & 21.74 \\
C2 & $251-750$ & Good & 13 & 56.52 \\
\hline
\end{tabular}

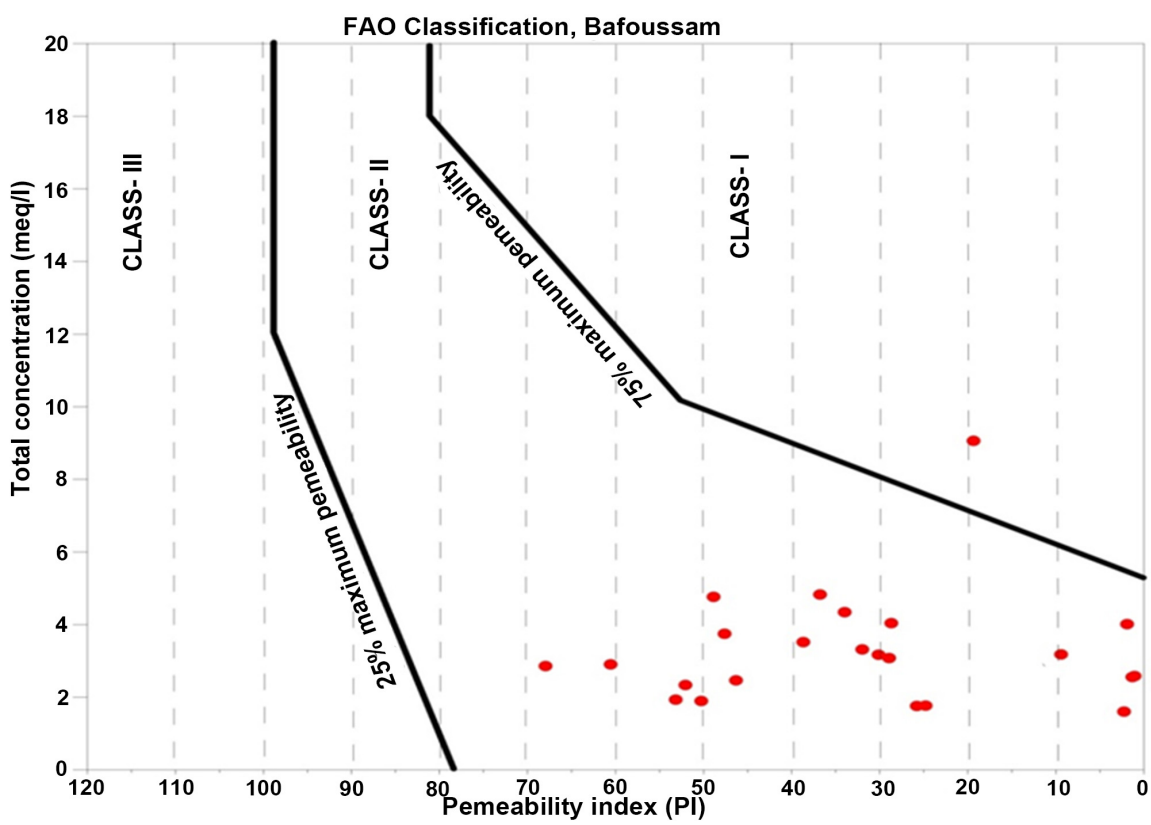

Figure 21. FAO classification of groundwater for irrigation indicating that the water is suitable for irrigation in the as the samples plot in class I and class II fields.

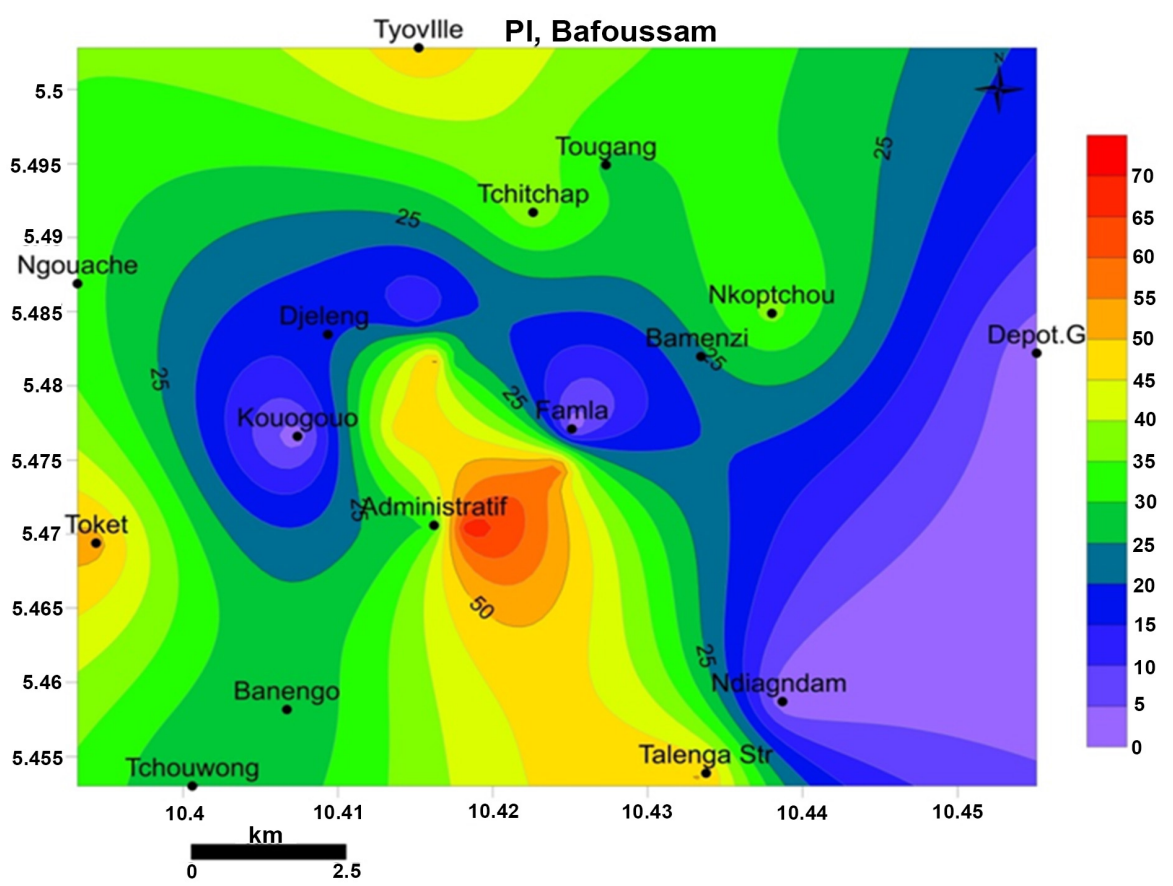

Figure 22. Spatial variation of permeability index; with high values at Administratif, Toket and Tyoville whereas low values are at Ndiangdam and Depot G. 


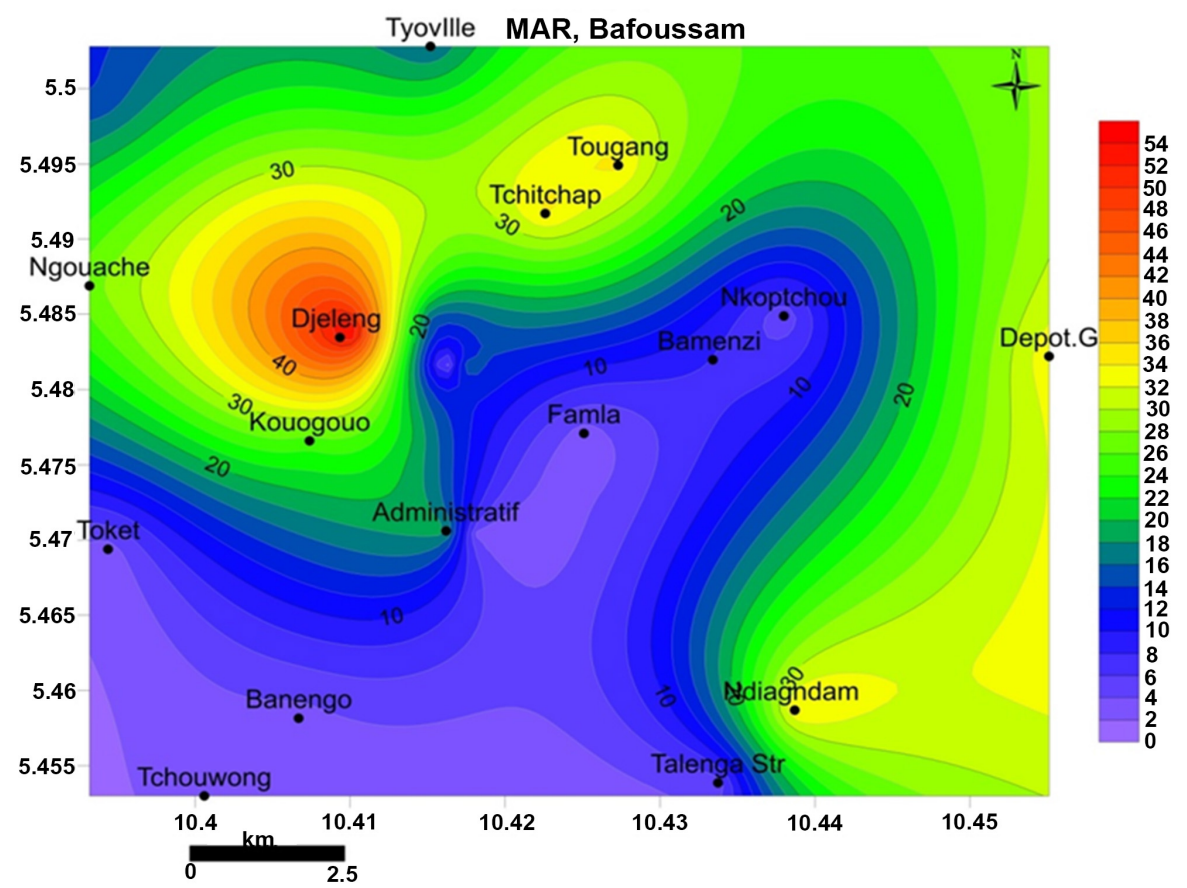

Figure 23. Spatial variation of Magnesium adsorption ratio; with high values at Djeleng, and Ngouache whereas low values are at Toket and Banengo.

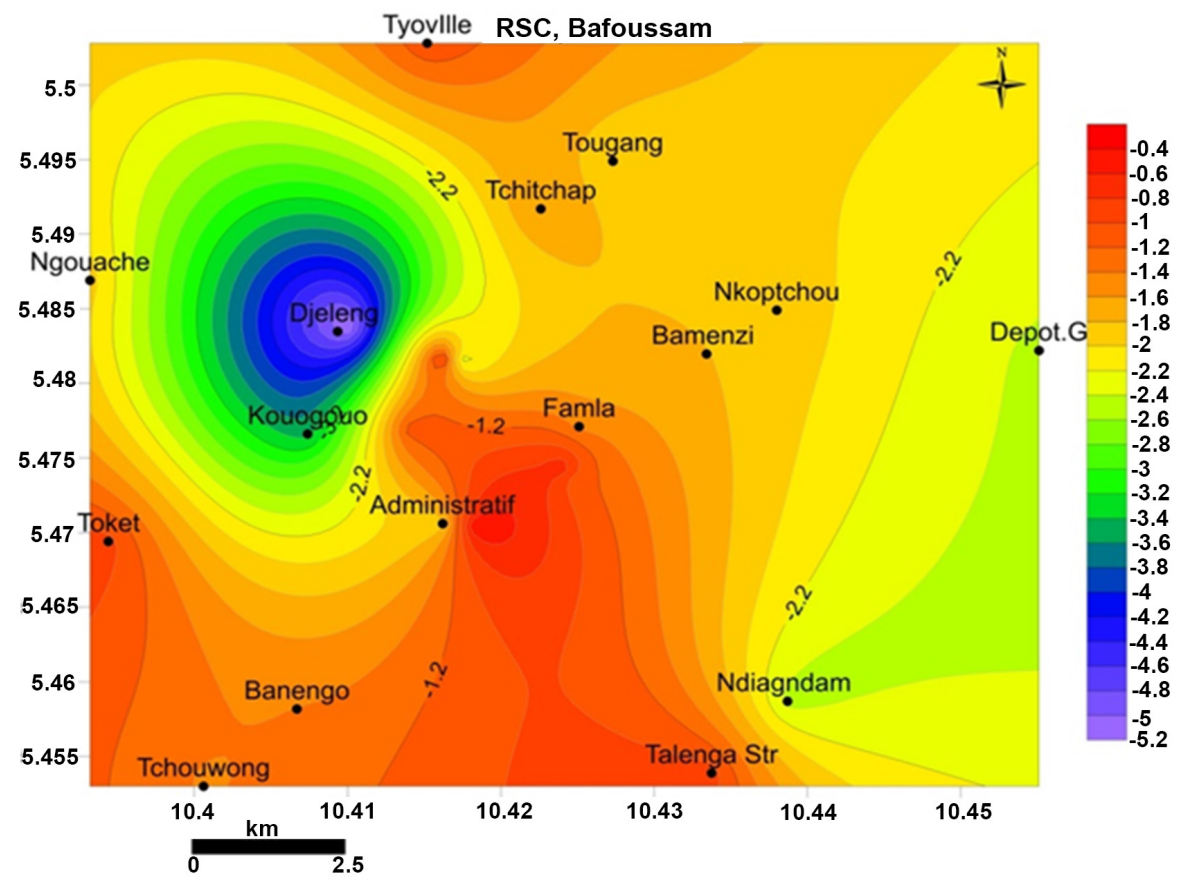

Figure 24. Spatial variation of Residual sodium carbonate with low values in all parts of Bafoussam.

\section{Discussion}

Groundwater contours are concentric around areas of high elevation and mimic the surface topography; indicative of topography controlled piston flow in a phreatic aquiferous formation. 


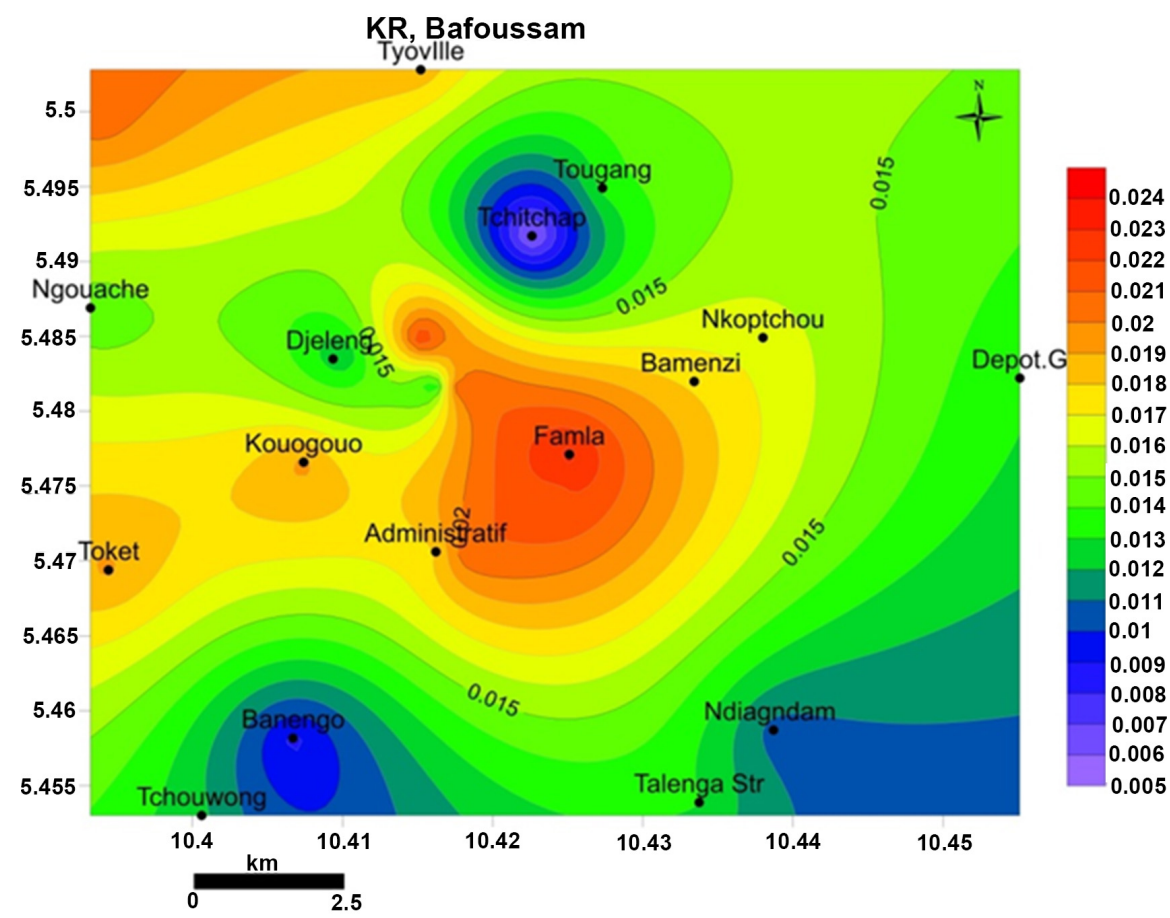

Figure 25. Spatial variation of Kelly's ratio; with relatively high values at Famla and Administratif whereas low values are at Banengo.

The results of laboratory analysis of groundwater together with hydrogeochemical tools have elucidated the processes and mechanisms affecting the groundwater chemistry in Bafoussam: Chemical weathering of rock-forming minerals is the factor influencing the processes of groundwater ions acquisition from the matrix of the aquiferous formation while ion exchange, dissolution and mixing are the mechanisms by which the groundwater equilibrates and develops its chemical character over its flow domain. The dominant water types are chloride and bicarbonate Ca-rich waters and $M g$-rich waters. The dominant Hydrogeochemical facies are; $\mathrm{Ca}-\mathrm{Mg}-\mathrm{HCO}_{3}$ and $\mathrm{Ca}-\mathrm{Mg}-\mathrm{Cl}-\mathrm{SO}_{4}$. All samples are 'fresh water' types with low values of EC and TDS.

The Ca-Mg- $\mathrm{HCO}_{3}$ hydrogeochemical facies is characteristic of freshly recharged groundwater that has equilibrated with $\mathrm{CO}_{2}$ and soluble carbonate minerals under open system conditions in the vadose zone in a single hydraulic phreatic aquiferous fractured rock formation; the Bafoussam granito-basaltic fractured rock aquifer.

The Bafoussam groundwater samples in this fractured rock aquifer belong to the bicarbonate group with significant amounts of calcium and magnesium cations. The Sodium and Potassium content can be attributed to the presence of clays in the geology resulting in the release of potassium and sodium ions from the argillaceous materials by ion-exchange processes between the formation waters and clay minerals.

The overall chemical qualities of ground water with respect to drinking standards are good with low TDS and are soft. 
Quantitative chemical analysis results reflect the dominant anions to be calcium and Magnesium and the dominant anions bicarbonate and chloride characteristic of the ion exchange and dissolution of granitoids and basaltic weathering products.

The $\mathrm{pH}$, electrical conductivity, total dissolved solids and temperature values of water samples are below guideline acceptable limits and variable with seasons indicative of phreatic aquifers [22].

RSC, SAR, SSP, MAR, PI and KR indicating the suitability of groundwater samples for irrigation is Excellent-to-Good in almost all cases. By these indices the groundwater quality of Bafoussam has been assessed for its agro-industrial suitability, found to neither be able to cause salinity hazards nor have an adverse effect on the soil properties and is thus suitable for irrigational purposes.

\section{Conclusions}

The sequence of the abundance of the major cations is $\mathrm{Ca}>\mathrm{Mg}>\mathrm{K}>\mathrm{Na}>\mathrm{NH}_{4}$; and anions are $\mathrm{HCO}_{3}>\mathrm{Cl}>\mathrm{SO}_{4}>\mathrm{NO}_{3}^{+}>\mathrm{HPO}_{4}^{2-}$.

Source rocks of ions in groundwater are the basalts and granitoids and these formations contribute overwhelmingly to the solute hydrogeochemistry.

The relationship between water composition and the main hydrogeochemical processes for ions acquisition is rock weathering.

$\mathrm{Ca}-\mathrm{HCO}_{3}, \mathrm{MgHCO}_{3}$ and $\mathrm{Ca}-\mathrm{Cl}$ are the water types and $\mathrm{Ca}-\mathrm{Mg}-\mathrm{Cl}-\mathrm{SO}_{4}$ and $\mathrm{Ca}-\mathrm{Mg}-\mathrm{HCO}_{3}$ hydrogeochemical facies.

Precipitation recharge and ion exchange are the hydrogeochemical processes determining the character of the groundwater.

Ionic ratio values for nitrate and sulfate are very low as such there are no serious anthropogenic contribution and no oxidation of sulphides.

The inorganic content of the groundwater in the granito-basaltic aquiferous formations in Bafoussam is of excellent to good quality for domestic use and suitable for agro-industrial purposes.

Recharge from precipitation exchanges ions with the weathered country rocks and mixes with regional flow in a generally south east north westerly direction by piston flow in the granito-basaltic aquiferous formations in Bafoussam.

There is a need for more detailed studies to determine aquifer characteristics: permeability, transmissivity and storativity, vertical and lateral regional extent of aquifer boundaries in this aquifer, groundwater pollution potentials for biological, organic and trace metals.

\section{Funding}

This research did not receive any specific grant from funding agencies in the public, commercial, or not-for-profit sectors.

\section{Acknowledgements}

We sincerely thank all the field workers and the private well owners for well construction data and access to wells. 


\section{Conflicts of Interest}

The authors declare no conflicts of interest regarding the publication of this paper.

\section{References}

[1] Divisional Delegation of the Ministry of Economy, Planning and Regional Development, MINEPAT (2016) Report on the State of Socioeconomic Development in the Mifi Division.

[2] Ezzati, M., Lopez, A.D., Rodgers, A. and Murray C.J.L. (2004) Comparative Quantification of Health Risks; Global and Regional Burden of Disease Attributable to Selected Major Risk Factors. World Health Organization, Geneva.

[3] Djouka-Fonkwé, M.L., Schulz, B., Schüssler, U., Tchouankoué, J.P. and Nzolang, C. (2007) Geochemistry of the Bafoussam Pan-African I- and S-type Granitoids in Western Cameroon. Journal of African Earth Sciences, 50, 148-167. https://doi.org/10.1016/j.jafrearsci.2007.09.015

[4] ISO (2006) Standard ISO 5667 1: Water Quality-Sampling-Part 1: Guidance on the Design of Sampling Programs and Sampling Techniques. International Organization for Standardization, Geneva.

[5] ISO (2003) Standard ISO 5667 3: Water Quality-Sampling-Part 3: Guidance on the Preservation and Handling of Water Samples. International Organization for Standardization, Geneva.

[6] ISO (2009) Standard ISO 5667-11: Water quality-Sampling-Part 11: Guidance on Sampling of Groundwaters. International Organization for Standardization, Geneva.

[7] American Public Health Association APHA (1995) Standard Methods for Examination of Water and Waste Water. American Public Health Association, American Water Works Association and Water Pollution Control Federation, Washington DC, USA.

[8] Hounslow, A.W. (1995) Water Quality Data: Analysis and Interpretation. Lewis Publishers CRC Press, New York, 397.

[9] Gibbs, R.J. (1970) Mechanisms Controlling World's Water Chemistry. Science, 170, 1088-1090. https://doi.org/10.1126/science.170.3962.1088

[10] Piper, A.M. (1944) A Graphic Procedure in the Geochemical Interpretation of Water Analysis. American Geophysical Union Transactions, 25, 914-928. https://doi.org/10.1029/TR025i006p00914

[11] Langguth, H.R. (1966) Groundwater verhaltisse in Bereiech Des VelberterSattles. Der Minister Fur Eraehrung. Land Wirtsch Forste Duesseldorf: NRW, 127.

[12] Durov, S.A. (1948) Classification of Natural Waters and Graphical Representation of Their Composition. Doklady Akademii Nauk SSSR, 59, 87-90.

[13] Lloyd, J.A. and Heathcote, J.A. (1985) Natural Inorganic Hydrochemistry in Relation to Groundwater: An Introduction. Oxford University Press, New York, 296.

[14] Wilcox, L.V. (1995) Classification and Use of Irrigation Waters, U.S.D. A Circular No. 960, Washington DC, 19.

[15] Kelley, W.P. (1940) Permissible Composition and Concentration of Irrigation Waters. Proceedings of the American Society of Civil Engineers, 66, 607-613.

[16] Paliwal, K.V. (1972) Irrigation with Saline Water. Monogram No. 2 (New Series). IARI, New Delhi, 198. 
[17] Todd, D.K. (1980) Ground Water Hydrogeology. Wiley International Edition, John Wiley and Sons, New York.

[18] Eaton, E.M. (1950) Significance of Carbonate in Irrigation Water. Soil Science, 69, 123-133. https://doi.org/10.1097/00010694-195002000-00004

[19] Richards, L.A. (1954) Diagnosis and Improvement of Saline Alkali Soils: Agriculture. Vol. 160, Handbook 60, USDA, Washington DC.

[20] Doneen, L.D. (1962) The Influence of Crop and Soil on Percolating Water. Biennial Conference on Groundwater Recharge, 156-163.

[21] Sisodia, R. and Moundiotiya, C. (2006) Assessment of the Water Quality Index of Wetland Kalakholake, Rajasthan, India. Journal of Environmental Hydrology, 14, $1-11$.

[22] World Health Organization (2017) Guidelines for Drinking-Water Quality. 4th Edition, Incorporating the First Addendum, Geneva.

[23] Lukas, E. (2003) Windows Interpretation for Hydrogeologists (WISH) Version 2 (Build 160). IGS-UFS, Bloemfontein.

[24] Sawyer, G.N. and McCarthy, D.L. (1967) Chemistry of Sanitary Engineers. 2nd Edition, McGraw Hill, New York, 518.

[25] Babaei, S.F. (2011) Evolution of a New Surface Water Quality Index for Karoon Catchment in Iran. Journal of Water Science and Technology, 64, 2483-2491. https://doi.org/10.2166/wst.2011.780

[26] United States Salinity Laboratory (1954) Diagnosis and Improvement of Saline and Alkali Soils. Agriculture Hand Book No. 60, Washington DC. 